\title{
1 Tomato CRABS CLAW paralogues interact with chromatin remodelling \\ 2 factors to mediate carpel development and floral determinacy \\ 4 Laura Castañeda ${ }^{1}$, Estela Giménez ${ }^{1}$, Benito Pineda ${ }^{2}$, Begoña García-Sogo ${ }^{2}$, Ana \\ 5 Ortiz-Atienza ${ }^{1}$, Rosa Micol-Ponce ${ }^{1,3}$, Trinidad Angosto ${ }^{1}$, Juan Capel ${ }^{1}$, Vicente \\ 6 Moreno $^{2}$, Fernando J. Yuste-Lisbona ${ }^{1}$, Rafael Lozano ${ }^{1}$ \\ $8 \quad{ }^{1}$ Centro de Investigación en Biotecnología Agroalimentaria (CIAIMBITAL). \\ 9 Universidad de Almería, 04120 Almería, Spain. \\ $10{ }^{2}$ Instituto de Biología Molecular y Celular de Plantas (UPV-CSIC). Universidad \\ 11 Politécnica de Valencia, 46022 Valencia, Spain. \\ $12{ }^{3}$ Present address: Instituto de Bioingeniería, Universidad Miguel Hernández, \\ 13 Campus de Elche, 03202, Elche, Spain.

15 Corresponding author: Prof. Rafael Lozano

16 Departamento de Biología y Geología (Genética),

17 Edificio CITE II-B, Universidad de Almería

18 Carretera de Sacramento s/n, 04120 Almería, Spain

19 Phone: +34) 950 015111; Fax: +34) 950015476

20 e-mail: rlozano@ual.es

22 Short title: SICRC genes trigger floral determinacy

24 The author(s) responsible for distribution of materials integral to the findings 25 presented in this article in accordance with the policy described in the Instructions 26 for Authors (https://academic.oup.com/plcell/pages/General-Instructions) is: Rafael 27 Lozano (rlozano@ual.es). 


\section{Abstract}

31 CRABS CLAW (CRC) orthologues play a crucial role in floral meristem (FM)

32 determinacy and gynoecium formation across angiosperms, key developmental

33 processes for ensuring successful plant reproduction and crop production. Here,

34 we revealed that the incomplete penetrance and variable expressivity of the carpel-

35 inside-carpel phenotype observed in flowers of the tomato fruit iterative growth (fig)

36 mutant is due to a lack of function of a homologue of the CRC gene, Solanum

37 lycopersicum CRCa (SICRCa). Likewise, a comprehensive functional analysis of

38 SICRCa and SICRCb paralogues, including Arabidopsis complementation

39 experiments, allowed us to propose that they operate as positive regulators of FM

40 determinacy by acting in a compensatory and partially redundant manner to

41 safeguard the proper formation of flowers and fruits. Furthermore, we provide the

42 first evidence for the role of putative CRC orthologues as members of the 43 chromatin remodelling complex that terminates floral stem cell activity by 44 repressing WUSCHEL expression. 


\section{Introduction}

47 Angiosperms are characterised by producing flowers as reproductive organs to 48 ensure their effective reproduction. From their outermost to the innermost whorls, 49 flowers typically consist of sepals, petals, stamens and carpels that are 50 sequentially generated from a pool of stem cells located in the floral meristems 51 (FM) (Krizek and Fletcher, 2005; Aichinger et al., 2012). Once a set number of 52 floral organs has been initiated, stem cell activity is arrested, and the FM is thereby 53 determined to form the female reproductive structure known as gynoecium. The 54 precise timing of this developmental event, also referred to as floral determinacy, is

55 a pivotal process that establishes a defined number of floral organs arising from 56 the FM (Sablowski, 2007; Sun et al., 2009; Sun and Ito, 2015).

In the model species Arabidopsis thaliana, the homeodomain transcription factor WUSCHEL (WUS) is responsible for the maintenance of the stem cell activity in the FM. The MADS-box transcription factor AGAMOUS (AG) plays a key role in the timing regulation of FM termination by repressing WUS expression (Laux et al., 1996; Mayer et al., 1998; Liu et al., 2011). AG turns off the stem cell maintenance programme involving transcriptional repression of WUS by different pathways; directly, by a mechanism that implicates chromatin remodelling and the recruitment

65 of the Polycomb Group (PcG) protein TERMINAL FLOWER2/LIKE 66 HETEROCHROMATIN PROTEIN1 (TFL2/LHP1) at the WUS locus (Liu et al., 67 2011; Guo et al., 2018), and indirectly through the transcriptional induction of two 68 key downstream targets, KNUCKLES (KNU) and CRABS CLAW (CRC), which act 69 through independent pathways to synergistically regulate WUS repression and ensure an adequate FM determination process (Payne et al., 2004; Sun et al., 71 2009, 2014, 2019; Yamaguchi et al., 2017, 2018). The KNU gene encodes a C2H2

72 zinc-finger protein whose expression is activated by AG, a process that requires a 73 time-delay induction regulated by epigenetic modification of histones at the KNU 74 locus (Sun et al., 2014). Once induced, KNU binds to the WUS promoter, which 75 causes the eviction of SPLAYED (SYD), a chromatin remodelling factor required 
76 for WUS activation, and mediates the subsequent deposition of H3K27me3 for

77 stable Polycomb-mediated repression of WUS (Kwon et al., 2005; Sun et al.,

78 2019). Furthermore, AG also positively regulates MINI ZINC FINGER2 (MIF2)

79 expression during flower development, which acts as an adaptor protein to form a

80 transcriptional repressor complex together with $\mathrm{KNU}$, the transcriptional

81 corepressor TOPLESS (TPL), and the chromatin remodelling protein HISTONE

82 DEACETYLASE19 (HDA19). Within this complex, MIF2 binds to the WUS locus,

83 leading to an epigenetically repression of WUS expression through histone 84 deacetylation (Kagale and Rozwadowski, 2011; Krogan et al., 2012; Bollier et al., 85 2018). Concurrently, the YABBY transcription factor CRC, a direct target of $A G$, 86 mediates auxin homeostasis and establishes auxin maxima during carpel primordia 87 initiation by repressing TORNADO2 (TRN2) and upregulating the auxin synthesis 88 gene YUCCA4 (YUC4). The proper auxin maxima mediated by CRC contributes to 89 the termination of FM cells proliferation through WUS repression and triggers the 90 subsequent gynoecium formation (Yamaguchi et al., 2017, 2018).

92 In tomato (Solanum lycopersicum L.), the molecular mechanisms underlying 93 SIWUS transcriptional regulation during floral development are of agronomic 94 interest, as mutations leading to a spatial and temporal expansion of its expression 95 domains in FMs result in flowers with extra carpels, which give rise to larger 96 multilocular fruits (Muños et al., 2011; van der Knaap et al., 2014; Xu et al., 2015; 97 Rodríguez-Leal et al., 2017; Yuste-lisbona et al., 2020). Likewise, alterations in 98 SIWUS transcriptional repression during flower development potentially lead to 99 indeterminate fruits, which makes floral determinacy a developmental process 100 closely related to fruit shape, an important fruit quality attribute as it influences 101 consumer's acceptance and postharvest handling. Recently, Bollier et al. (2018) 102 have proposed a conserved molecular mechanism regulating FM determinacy in 103 Arabidopsis and tomato. Thus, the interaction of tomato MIF2 and KNU 104 orthologues, INHIBITOR OF MERISTEM ACTIVITY (SIIMA) and SIKNU, allows for 105 the recruitment of tomato TPL and HDA19 orthologues, SITPL1 and SIHDA1, to 106 form a transcriptional repressor complex at the SIWUS locus. In the present study, 
107 we provide novel insights into the genetic and molecular mechanism involved in

108 FM determinacy and carpel development. Through combining mapping-by-

109 sequencing, RNA interference (RNAi) and CRISPR/Cas9 techniques, we revealed

110 that the carpel-inside-carpel phenotype observed in fruit iterative growth (fig)

111 mutant plants is due to the lack of function of the S. lycopersicum CRC homologue

112 SICRCa. Furthermore, we carried out a detailed functional analysis of tomato CRC

113 paralogues, SICRCa and SICRCb, which allowed us to uncover for the first time

114 the role of putative CRC orthologues as members of the molecular regulatory

115 network that epigenetically represses WUS through histone deacetylation to

116 ensure the proper termination of floral stem cell activity. 


\section{Results}

\section{The fig mutation impairs carpel determinacy}

123 The fruit iterative growth (fig) mutant was isolated from the screening of a collection

124 of $\mathrm{T}_{1}$ segregating T-DNA lines generated from the tomato cultivar P73 (Pérez-

125 Martín et al., 2017). A detailed phenotypic analysis revealed that the vegetative 126 development of fig mutant plants was indistinguishable from that in wild-type (WT) 127 ones, whereas significant differences were observed during flower and fruit 128 development (Fig. 1a-c and Supplementary Table 1). fig flowers developed an 129 elevated number of organs in all whorls, being this increase more evident in 130 carpels. Thus, fig ovaries are composed of numerous carpels that lead to 131 anomalous fruits which show secondary fruit structures growing in an

132 indeterminate way that appeared from the inside of the principal fruit (Fig. 1a-C and 133 Supplementary Table 1).

135 The observed phenotypic segregation (39 WT: 15 fig) was consistent with a 136 monogenic recessive inheritance of the fig mutation $\left(x^{2}=0.22 ; P=0.64\right)$. However, 137 the phenotype of fig plants showed incomplete penetrance and variable 138 expressivity, as a gradation of phenotypes was displayed within the same mutant 139 plant, even within the same inflorescence. Thereby, variable flower and fruit 140 phenotypes were observed in fig plants, which were classified as WT-like 141 (indistinguishable from WT), weak and severe indeterminate phenotypes (Fig. 1a-c 142 and Supplementary Fig. 1), being the average production of fruits with said severe 143 fig phenotype close to $50 \%$ per mutant plant (Fig. 1d).

145 A scanning electron microscopy (SEM) study in developing flowers revealed that 146 the first visible anomalies could be detected at floral stage 5 as estimated by floral 147 bud size (Brukhin et al., 2003). At this stage, the carpels emerge, and the ovary 148 cavities become visible showing abnormal carpel structures in both weak and 149 severe fig flowers (Fig. 1f,g). Later, at stage 8, WT pistils are formed by 3-4 carpels 
150 (Fig. 1e), whereas an increased number of carpels are observed in fig flowers 151 resulting in incomplete fused pistils (Fig. 1f,g and Supplementary Fig. 1). Moreover, 152 histological sections of flowers at anthesis day showed that fig pistils have shorter 153 and thicker styles and are composed of numerous carpels that grow one inside 154 another, which strongly suggests that fig mutation affects carpel determinacy. 155 These differences were more accentuated in severe fig flowers (Fig. 1c,g), 156 producing ovaries threefold bigger than the WT ones at anthesis day stage (Fig. 157 1a,e). As a result of this variable range of fig flower phenotypes, we could observe 158 both weak fig fruits producing secondary fruit structures only visible inside the fruit 159 (Fig. 1b), and severe fig fruits where these secondary fruit structures emerged from 160 inside and were visible outside the fruit (Fig. 1c and Supplementary Fig. 1). Despite 161 such abnormalities and although more extreme fig fruits produced fewer seeds, fig 162 mutants gave rise to viable seeds. 

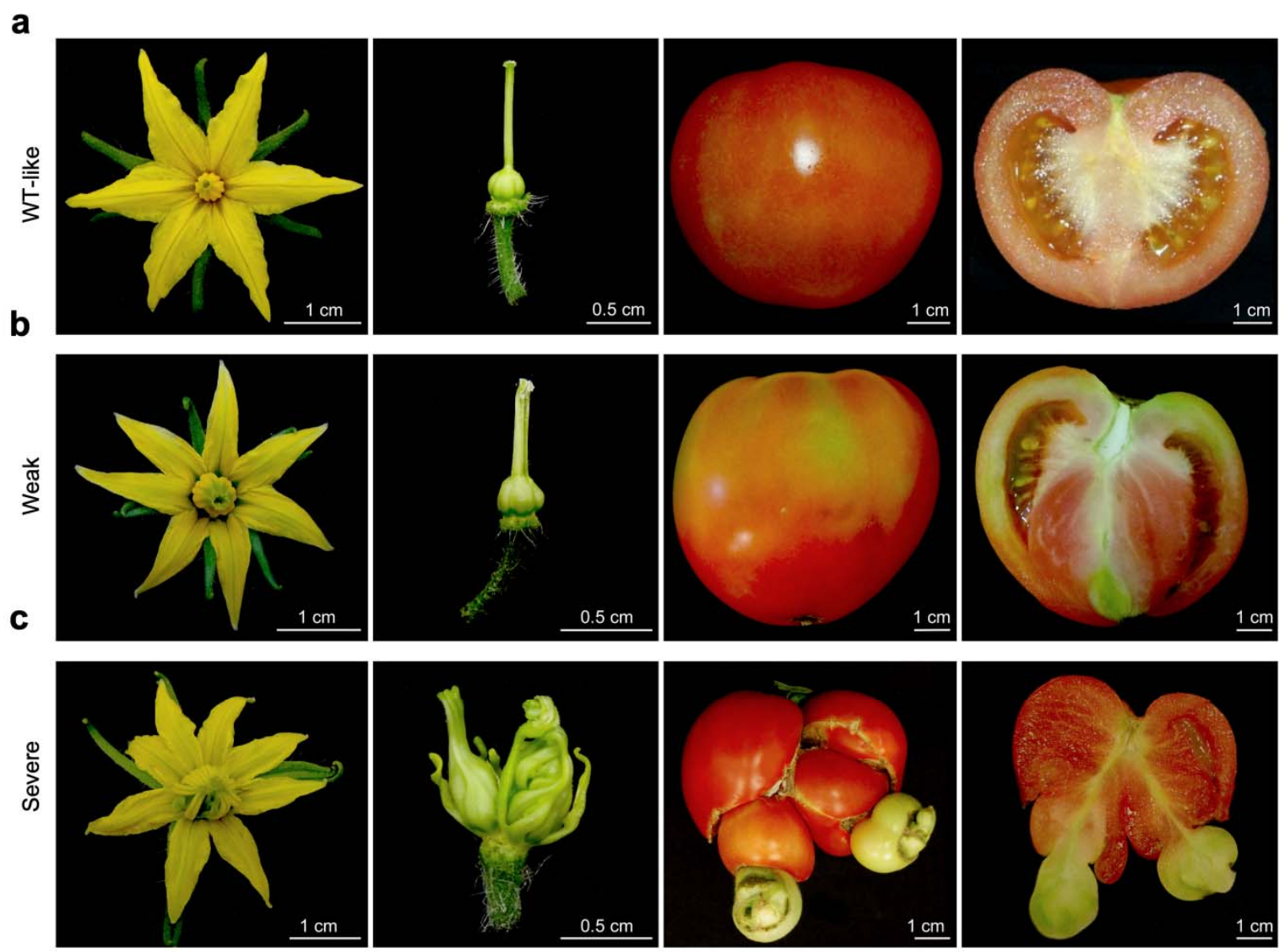

d

$\mathbf{e}$

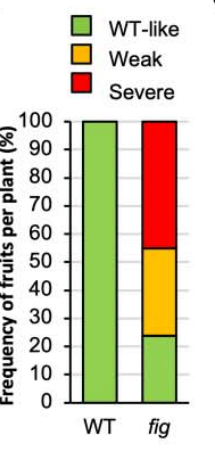
$N / n=50 / 108852 / 905$

f WT-like

Stage 5 $A D$

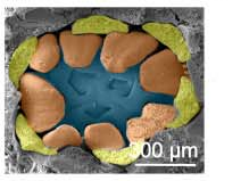

Stage 8

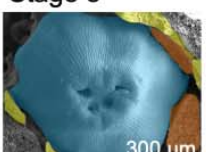

$300 \mu \mathrm{m}$

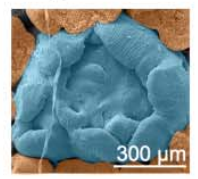

Stage 8

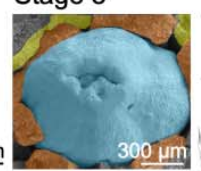

g

$A D$

Severe

Stage $5 \quad A D$

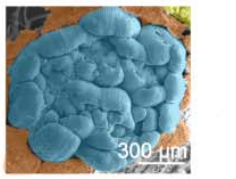

Stage 8
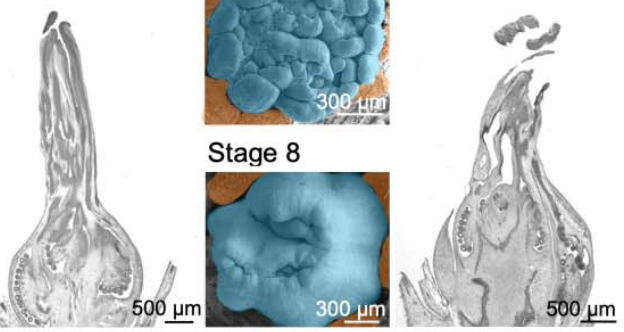

165 Fig. 1 | Phenotypic characterisation of the fig mutant. a-c, Representative fig

166 flowers, pistils, closed fruits, and longitudinal open fruits (from left to right) showing

167 WT-like (identical to wild-type, WT) (a), weak (b) and severe (c) phenotypes. d,

168 Percentage of different types of fruits (WT-like, weak or severe) produced by WT

169 (cv. P73) and fig plants. N, number of plants evaluated; n, number of fruits

170 harvested. e-g, Scanning electron microscopy images of flowers at stages 5 and 8

171 of floral development, and histological sections of flowers at anthesis day (AD)

172 stage exhibiting WT-like (e), weak (f) and severe (g) phenotypes. Sepals were 
173 removed in samples at stage 5 whereas only the carpels were maintained in

174 developing flowers at stage 8. Petals are coloured in yellow, stamens in orange, 175 and carpels in blue.

177 FIG encodes a homologue of the Arabidopsis CRC gene

179 Although the fig mutant was isolated from a T-DNA insertional mutant collection, 180 molecular analysis performed by Southern blot and PCR assays revealed that the 181 fig mutation was not associated with a T-DNA insertion (Supplementary Fig. 2), 182 suggesting that somaclonal variation produced during the in vitro culture process is 183 responsible for the mutant phenotype. To identify the causative mutation 184 underlying the fig phenotype, we performed a mapping-by-sequencing strategy 185 using an $\mathrm{F}_{2}$ mapping population derived from crossing fig with the wild tomato $S$. 186 pimpinellifolium (accession LA1589). A total of $783 F_{2}$ plants were scored for ovary 187 and fruit development, from which 212 plants produced fused carpels and 188 indeterminate fruits. The phenotypic segregation observed in this interspecific $F_{2}$ 189 progeny (571 WT: 212 fig) was consistent with a monogenic recessive inheritance 190 pattern of the fig mutation $\left(x^{2}=1.80, P=0.18\right)$. To perform mapping-by191 sequencing, we conducted genome sequencing of two DNA pools containing 50 192 WT and 25 fig $\mathrm{F}_{2}$ plants with the most strongly indeterminate phenotype. Genome193 wide analysis of the allele frequencies revealed a region encompassing the 194 centromere of chromosome 1 (2.4-70 Mb) with a strong bias towards tomato 195 reference alleles (Fig. 2a). The variant analysis encompassing the candidate 196 genomic region led to the identification of three SNPs mapping at the fourth intron 197 of the Solyc01g010240 gene, which also showed a marked decrease in the 198 sequence coverage of the mutant pool compared to other coding and non-coding 199 regions of this gene. To confirm the polymorphisms identified, PCR and Sanger 200 sequencing analyses with primers flanking the region containing these SNPs were 201 performed in WT and fig plants. The results showed that the PCR product from fig 202 genomic DNA, which included the fourth intron of the Solyc01g010240 gene, not 203 only contained the three SNPs mentioned above, but it was also larger than 
204 expected. Sequence analysis verified that a DNA fragment of $367 \mathrm{bp}$ was inserted 205 at position 5,053,687 on the chromosome 1 of the mutant genome (Assembly 206 SL4.0), interrupting the intron sequence between the Solyc01g010240 exons 4 and 2075 (Fig. 2b). This insertion shares a sequence identity of $86 \%$ with the Long 208 Terminal Repeat (LTR) copy placed between 6,336,872 and 6,337,201 positions 209 on the chromosome 10 (Assembly SL4.0), which may have acted as a 210 transposable element. Co-segregation analysis performed in the $F_{2}$ segregating 211 population showed that the 212 fig mutant plants were homozygous for the LTR 212 insertion, whereas 393 and 178 phenotypically WT plants were hemizygous or 213 lacked the $367 \mathrm{bp}$ insertion, respectively. Hence, results indicate that the fig 214 phenotype co-segregated with the LTR insertion at the fourth intron of the 215 Solyc01g010240 gene.

217 Despite the fact that the $367 \mathrm{bp}$ insertion occurs at a non-coding region, we 218 considered the Solyc01g010240 gene as a strong candidate to be responsible for 219 the fig phenotype, since it encodes a homologue of the Arabidopsis CRC gene, 220 SICRCa, a putative transcription factor of the YABBY gene family that has been 221 described as a key gene involved in the regulation of carpel and nectary 222 development, as well as in FM determination (Alvarez and Smyth, 1999; Bowman 223 and Smyth, 1999). To determine the effect of the LTR insertion on SICRCa 224 expression, cDNA cloning and qRT-PCR expression analyses were performed. 225 Sequence analysis of WT and mutant cDNAs revealed that both were identical; 226 however, mutant plants showed significantly reduced SICRCa expression levels in 227 floral buds at developmental stages 0-6 and 7-12 (Fig. 2c). Thus, the 367 bp 228 insertion in the fourth intron of SICRCa reduced its transcript level to >2-fold 229 although still allowed the production of WT SICRCa mRNA, suggesting that this 230 intron may contain a transcriptional regulatory element critical for the maintenance 231 of the SICRCa spatio-temporal expression pattern.

233 To corroborate whether the lack of SICRCa function is responsible for the fig 234 phenotype, we generated RNAi-mediated knockdown lines with reduced levels of 
235 SICRCa transcripts in the cultivar P73 genetic background. Four independent first236 generation ( $\left.T_{0}\right)$ RNAi SICRCa diploid lines were evaluated, which showed >2-fold 237 decrease in SICRCa expression in floral buds at stage 0-6 (Fig. 2d). These 238 reduced SICRCa transcript levels led to the development of flowers with 239 supernumerary carpels that produced indeterminate fruits (Fig. 2e). As happened 240 with the fig mutation, RNAi SICRCa lines displayed a variable range of flower 241 phenotypes, resulting in the development of fruits with WT-like, weak and severe 242 indeterminate phenotypes (Fig. 2e,f). Furthermore, we engineered knockout 243 mutations by using the CRISPR/Cas9 system with a single guide RNA targeting 244 the second exon of the SICRCa gene (Fig. $2 \mathrm{~g}$ ). We assessed four independent $\mathrm{T}_{0}$ 245 diploid lines (CR-s/crca) that were biallelic for edited knockout alleles (Fig. 2g). The 246 CR-s/crca lines developed flowers and fruits which undoubtedly resembled the 247 phenotype observed in fig mutants, and they also produced a wide diversity of 248 flower and indeterminate fruit phenotypes, most of them classified as severe (Fig. $2492 \mathrm{f}, \mathrm{h})$. Thereby, although there were differences in the percentages of fruits with 250 severe mutant phenotype, both knockdown (RNAi) and knockout (CRISPR/Cas9) 251 alleles resulted in similar phenotypes with incomplete penetrance and variable 252 expressivity, indicating that any deficiency in SICRCa function might lead to a loss 253 of FM determinacy. Taken together, all these results strongly support that the 367 254 bp insertion in the fourth intron of the SICRCa gene is responsible for the 255 alterations in carpel and fruit development observed in the fig mutant. 
a

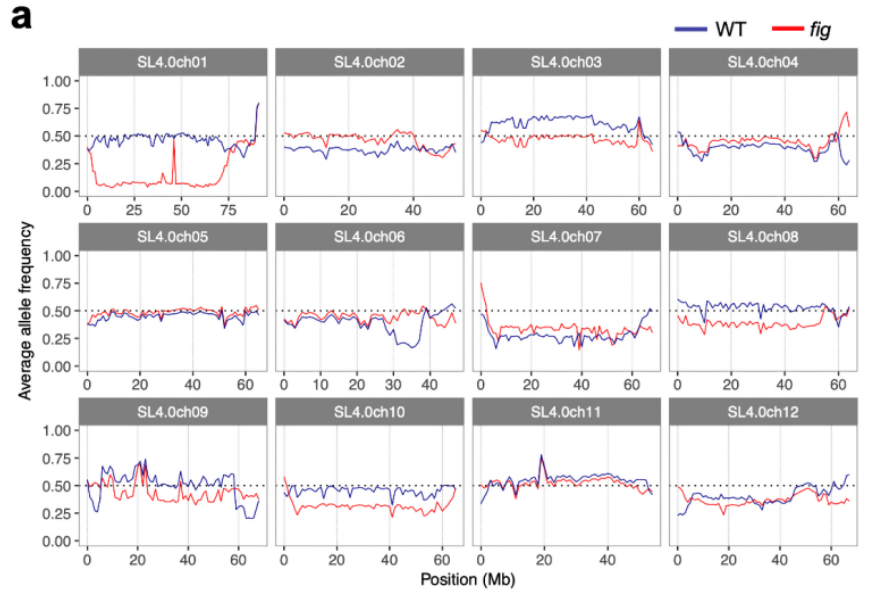

d

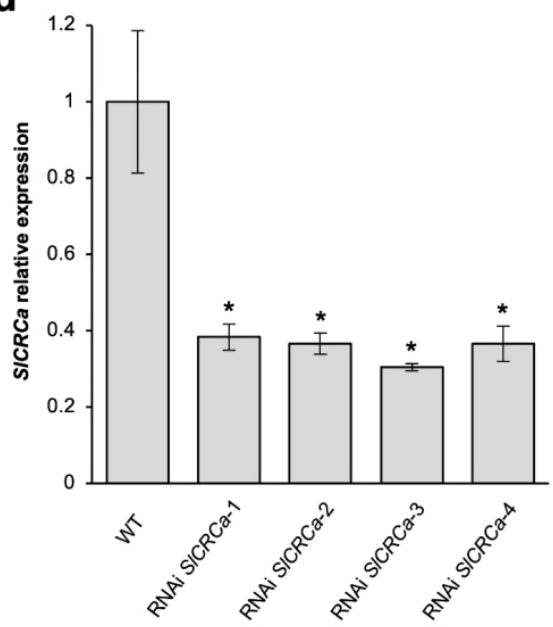

g

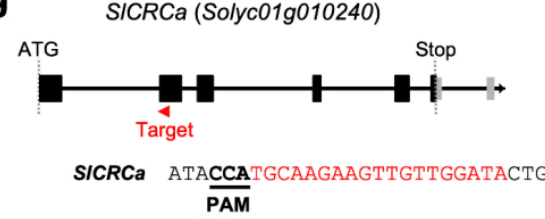

CR-slcrca-1 a1 ATACCATGCA----------ATACTG

CR-s/crca-

a2 ATA

CR-sica-3

a1 ATACCATG-AAGAAGTTGTTGGATACT

a2 ATACCATGC-AGAAGTTGTTGGATACTG

CR-s/crca-3

a1 ATACCATGCA----GTTGTTGGATACTG

a2 ATA

CR-slcrca-4

a1 ATACCAT--AAGAAGTTGTTGGATACTG

a2 ATACCATGC-AGAAGTTGTTGGATACTG e
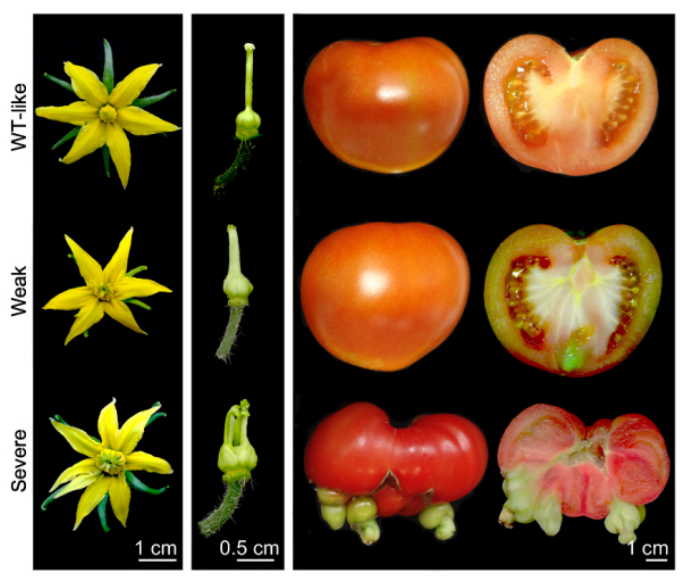

f

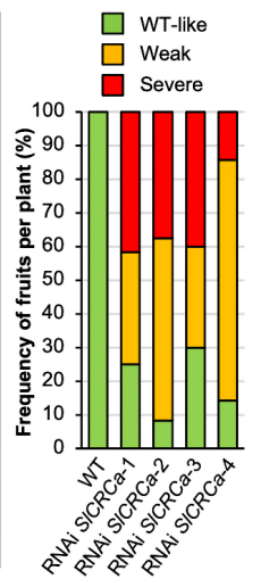

h
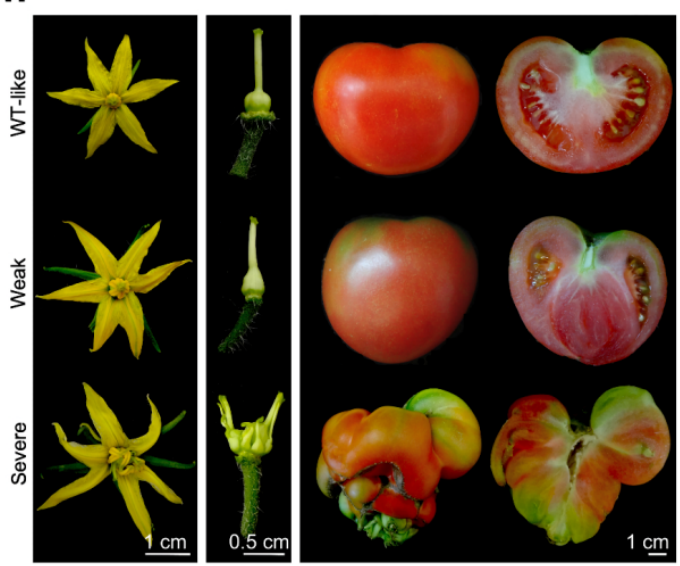

i

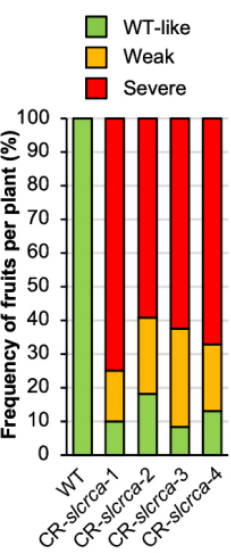

258 Fig. 2 | FIG encodes a homologue of the Arabidopsis CRC gene. a, Distribution

259 of the average allele frequency of wild-type (WT; blue line) and fig (red line) pools

260 grouped by chromosomes. b. Schematic representation of the SICRCa gene

261 (introns are represented by black lines, coding and UTRs are in black and grey 
262 boxes, respectively) and the 367 bp sequence inserted at the fourth intron in fig 263 mutants. c, Relative expression of SICRCa in WT and fig flowers at different stages

264 of floral development. FB0-6, floral buds from stages 0 to 6; FB7-12, floral buds 265 from stages 7 to 12; PA, flowers at pre-anthesis stage; AD, flowers at anthesis 266 stage; $A D+10$, flowers 10 days after anthesis stage. d, SICRCa transcripts 267 quantification of flowers at stage FBO-6 from RNAi SICRCa and WT lines. e, 268 Representative RNAi SICRCa flowers, pistils and fruits displaying WT-like, weak 269 and severe mutant phenotypes. f, Percentage of different types of fruits harvested 270 from WT and To RNAi SICRCa plants. g, CRISPR/Cas9-s/crca (CR-slcrca) alleles 271 identified by cloning and sequencing PCR products from the SICRCa targeted 272 region from four $\mathrm{T}_{0}$ CRISPR/Cas9 plants. Black bold and underlined letters indicate 273 protospacer-adjacent motif (PAM) sequences, blue dashed lines show InDel 274 mutations, blue letter and arrow indicate an insertion sequence. $\mathbf{h}$, Representative 275 CR-slcrca flowers, pistils and fruits exhibiting WT-like, weak and severe 276 phenotypes. i, Percentage of different types of fruits harvested from WT and $\mathrm{T}_{0}$ 277 CR-slcrca plants. In c,d data are means \pm standard deviations (SD) of three 278 biological and two technical replicates. A two-tailed, two-sample Student's $t$ test 279 was performed, and significant differences are represented by asterisks: *, $P<$ $280 \quad 0.01 ;{ }^{* *}, P<0.001 ;$ ns, no statistically significant differences.

282 SICRCa expression is restricted to carpels

284 To better understand the function of SICRCa, we monitored its expression pattern 285 in several vegetative and reproductive tissues. As expected from the phenotype of 286 fig mutant plants, SICRCa expression was mainly restricted to flowers during early 287 developmental stages. Thus, the higher expression level of SICRCa was found in 288 floral buds at stages 0-6 (Fig. 3a). We next performed an RNA in situ hybridisation 289 analysis in young flowers to further examine the temporospatial expression 290 patterns of SICRCa. This analysis revealed that SICRCa transcripts were 291 accumulated specifically and uniformly when carpel primordia were initiated at 292 floral stage 3 (Fig. 3b), and persisted in these primordia at floral stage 6, when 
293 carpels were growing up and the primordium of placenta emerged (Fig. 3c). At

294 floral stage 8, SICRCa expression was located at the adaxial surface on the base

295 of the ovary walls, as well as in the most distal cells of the developing gynoecium

296 which would give rise to the style and the stigma (Fig. 3d). However, SICRCa

297 mRNA was not detected in the ovary walls at floral stage 9, and its expression

298 remained in the distal part of the gynoecium (Fig. 3e). At later stages of floral

299 development, there was no evidence of detectable SICRCa transcripts.

301 RNA sequencing (RNA-seq) was next performed on WT and fig floral buds at

302 developmental stages 0-6 to gain insight into the functional role of SICRCa during

303 flower development. This analysis identified 2115 differentially expressed genes

304 (DEGs) in the fig mutant as compared to WT (false discovery rate adjusted P-value

$305<0.05)$, in which 978 were up-regulated and 1137 were down-regulated

306 (Supplementary Dataset 1), suggesting that decreasing SICRCa expression

307 considerably affected the transcriptome of fig floral buds. To investigate the

308 functions of differentially expressed genes, we applied a Gene Ontology (GO) term

309 enrichment analysis, which revealed 29 and 36 overrepresented GO terms for up-

310 and down-regulated DEGs, respectively (Supplementary Dataset 2). For biological

311 process, GO terms belonging to response to stimulus (GO:0050896) were enriched

312 in both groups of DEGs. However, biological processes related to reproduction

313 (GO:0000003), reproductive process (GO:0022414), developmental process

314 involved in reproduction (GO:0003006), reproductive structure development

315 (GO:0048608) and flower development (GO:0009908) were enriched among up-

316 regulated DEGs. With regard to molecular function, GO terms involved in protein

317 binding (GO:0005515) were enriched in both up- and down-regulated DEGs. In

318 addition, transporter activity (GO:0005215) and transcription factor activity,

319 sequence-specific DNA binding (GO:0003700) terms were enriched in up-

320 regulated DEGs (Supplementary Dataset 2 and Supplementary Fig. 3).

322 Among the up-regulated DEGs annotated with the reproductive structure 323 development (GO:0048608) and flower development (GO:0009908) terms, we 
324 found the tomato homologues of the Arabidopsis PIN-FORMED auxin efflux carrier 325 (Solyc03g118740, Solyc04g007690 and Solyc05g008060) and the AUXIN 326 RESPONSE FACTOR transcription factor (Solyc09g007810 and Solyc12g042070) 327 families, as well as the tomato homologues of the floral homeotic genes 328 APETALA2 (Solyc02g064960), APETALA3 (Solyc04g081000), FRUITFULL 329 (Solyc03g114830) and SEPALLATA4 (Solyc03g114840), the latter of which is also 330 involved in the determination of FM (Ditta et al., 2004). Within this group of genes, 331 we also found the homologue of the Arabidopsis HECATE3 (Solyc11g005780), 332 which encodes a bHLH transcription factor regulating the development of female 333 reproductive tissues. Thus, the overexpression of HECATE genes causes the 334 production of ectopic stigmatic tissue (Gremski et al., 2007). Remarkably, a second 335 homologue of the Arabidopsis CRC gene (SICRCb, Solyc05g012050) was 336 moreover included in this group of up-regulated DEGs. In summary, RNA-seq 337 analysis revealed that several genes involved in reproductive developmental 338 process and gynoecium formation were affected by the lack of function of SICRCa 339 gene. 

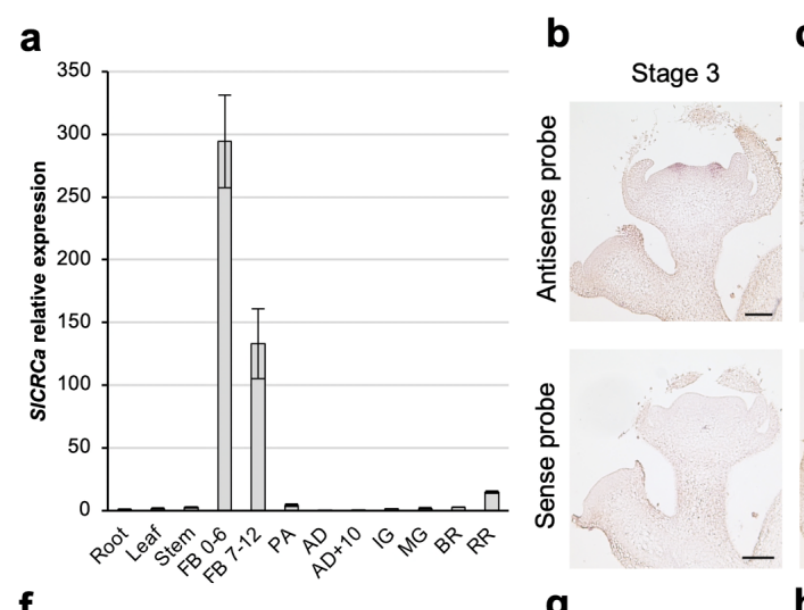

f
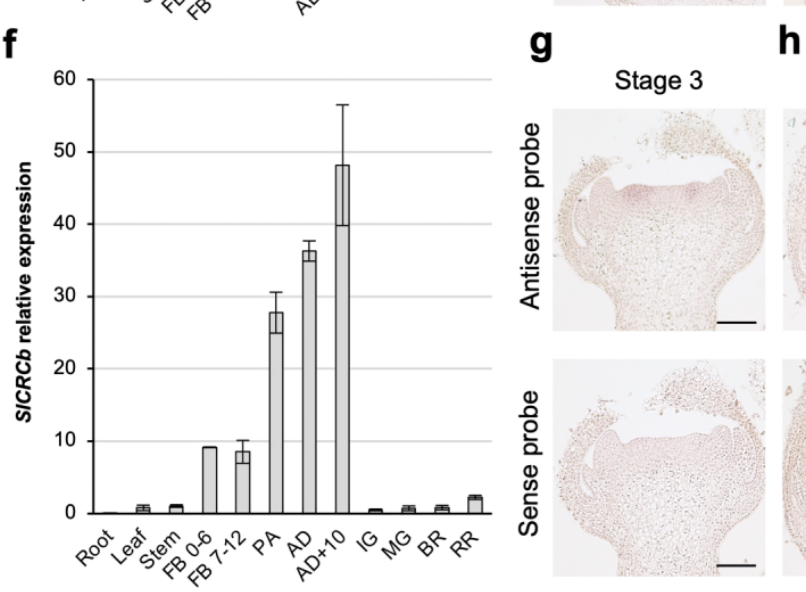

C Stage 6

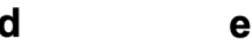

Stage 8

Stage 9
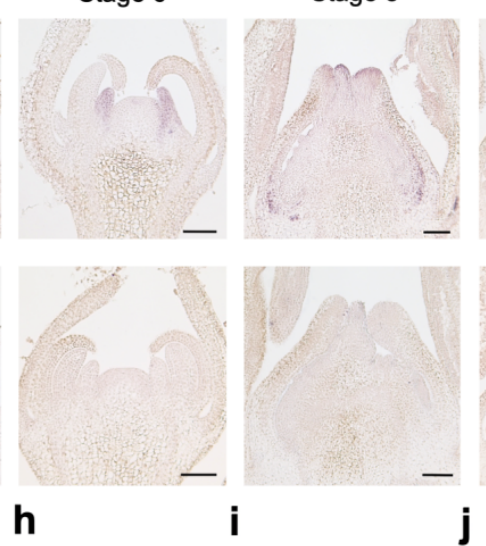

Stage 6

Stage 8

Stage 9

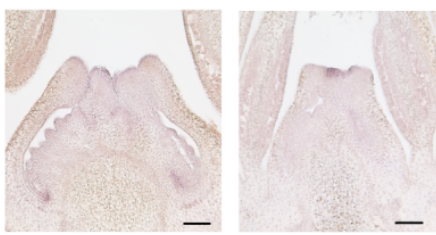

342 Fig. 3 | Dynamic expression of SICRCa and SICRCb genes. a, Relative

343 expression of SICRCa in different developmental tissues and stages of wild-type

344 (WT) flowers. b-e, In situ mRNA hybridisation of SICRCa using antisense or a

345 sense probes in histological sections of WT flowers at different developmental

346 stages: stage 3 (b), stage 6 (c), stage 8 (d) and stage 9 (e). f, Relative expression

347 of SICRCb in different developmental tissues and stages of WT flowers. g-j, In situ

348 mRNA hybridisation of SICRCb using antisense or a sense probes in histological

349 sections of WT flowers at different developmental stages: stage $3(\mathbf{g})$, stage $6(\mathbf{h})$, 350 stage 8 (i) and stage 9 (j). In a,f data are means \pm standard deviations (SD) of

351 three biological and two technical replicates. FB0-6, floral buds from stages 0 to 6 ;

352 FB7-12, floral buds from stages 7 to 12; PA, flowers at pre-anthesis stage; $A D$,

353 flowers at anthesis stages; $A D+10$, flowers 10 days after anthesis stage; IG;

354 immature green fruit; $M G$, mature green fruit; $B R$, breaker fruit; $R R$, mature red

355 fruit. In $\mathbf{b}-\mathbf{e}, \mathbf{g}-\mathbf{j}$ scale bars represent $100 \mu \mathrm{m}$. 
Knockout mutations in SICRCb mimic fig mutant phenotype

359 While the Arabidopsis genome carries one copy of the CRC gene, an ancestral 360 duplication, which likely happened before the Solanaceae diversification, 361 generated two paralogues, CRCa/CRCb, whose retention across Solanaceae 362 genomes suggests functional relevance (Phukela et al., 2020). Sequence analysis 363 of the Arabidopsis CRC (181 aa) and the tomato SICRCa (173 aa) and SICRCb 364 (160 aa) putative amino acid sequences displayed that CRC has an identity of 64 365 and $68 \%$ to SICRCa and SICRCb, respectively; while SICRCa shows $73 \%$ identity 366 to the aminoacidic sequence of SICRCb. Furthermore, the study of microsynteny 367 conservation between genomic blocks harbouring CRC genes in Arabidopsis and 368 tomato showed that the Arabidopsis genomic region containing CRC was more 369 closely related to that from tomato which bears SICRCb rather than SICRCa 370 (Supplementary Fig. 4). To gain further insight into the role of the SICRCb gene, 371 we examined the SICRCb spatio-temporal expression by qRT-PCR and in situ 372 hybridisation. As with SICRCa, a specific expression in reproductive tissues was 373 observed for SICRCb (Fig. 3a,f), although SICRCb transcripts were detected 374 throughout all floral development stages, from floral buds at developmental stages 375 0-6 to flowers at 10 days post anthesis (Fig. 3f), whereas SICRCa expression was 376 mainly detected at first stages of the floral bud development (Fig. 3a). Even though 377 SICRCa and SICRCb showed differential temporal expression patterns, in situ 378 hybridisation studies in developing flowers revealed that SICRCb shows an 379 identical spatial expression profile to SICRCa. Thus, SICRCb transcript 380 accumulation was first localised at floral stage 3 in carpel primordia (Fig. $3 \mathrm{~g}$ ), 381 where it continued until floral stage 6 (Fig. 3h). As the development progressed to 382 floral stage 8, SICRCb expression was detected in the ovary walls and the distal 383 regions of the developing gynoecium (Fig. 3i), where its expression remained 384 restricted at floral stage 9 (Fig. 3j).

386 The incomplete penetrance and variable expressivity of mutations at the SICRCa 387 locus, as well as the overlapping SICRCa and SICRCb spatial expression patterns 
388 at the initiation of gynoecium development, led to hypothesised that a partial 389 redundancy of tomato CRC paralogues may exist. To test this hypothesis, we 390 generated knockout mutations by using CRISPR/Cas9 system with a single guide 391 RNA targeting the second exon of the SICRCb gene (Fig. 4a). We evaluated four 392 independent $T_{0}$ diploid lines (CR-s/crcb) homozygous or biallelic for edited 393 knockout alleles (Fig. 4a). The CR-slcrcb lines produced flowers with ovaries 394 composed of numerous carpels growing inside one another, which led to 395 anomalous fruits similar to those observed in fig mutants, RNAi SICRCa and CR396 slcrca lines (Fig. 4b). CR-slcrcb lines also produced a wide diversity of flower and 397 indeterminate fruit phenotypes, although the presence of fruits with weak or severe 398 mutant phenotypes did not exceed 50\%, being the percentages of fruits with 399 severe mutant phenotype close to 15\% (Fig. 4c). These results revealed that 400 SICRCa and SICRCb could have partially redundant roles in FM determinacy.

402 Taking into account that RNA-seq results showed that SICRCb was up-regulated in 403 fig floral buds, the question arose as to whether a compensatory mechanism 404 between tomato CRC paralogues may be involved in gynoecium determination. 405 For that purpose, the expression of SICRCa and SICRCb genes was quantified in 406 reproductive tissues of slcrcb and slcrca CRISPR/Cas9 null mutants, respectively. 407 SICRCb was differentially up-regulated in CR-s/crca floral buds at developmental 408 stages 0-6 (Fig. 4d). SICRCb was also up-regulated in CR-slcrca from floral buds 409 to anthesis day during floral development; whereas SICRCa transcripts were up410 regulated in CR-slcrcb floral buds at developmental stages 0-6 (Fig. 4d). 411 Additionally, we generated CR-slcrca:slcrcb double mutant plants to further dissect 412 the relationship between tomato CRC paralogues. Remarkably, the simultaneous 413 loss of function of SICRCa and SICRCb resulted in homeotic alterations affecting 414 carpel development as the shape of some of the cells of which they were 415 composed attained a stamen-like nature (Fig. 4e and Supplementary Fig. 5). CR416 slcrca:slcrcb flowers produced stamen-like carpels in a reiterating pattern 417 exclusively affecting the fourth whorl, thus giving rise to fruits with a severe 418 indeterminate phenotype (Fig. 4f,g), which in all cases was considerably more 
419 severe than in either CR-slcrca or CR-slcrcb single mutant plants. Hence, complete

420 penetrance and invariable expressivity were found when both tomato paralogues

421 lost their functions.

422

a

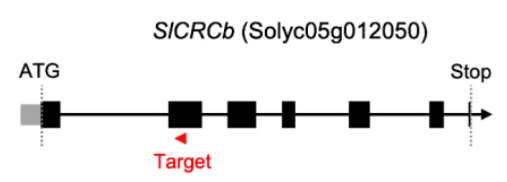

SICRCb ATTCCATACAAGAGGCTAATGGATACTGT CR-s/crcb-1 ATTCCATACA---GGCTAATGGATACTGT
a2 ATTCCATACAAGAGGCTAATGGATACTGT CR-s/crcb-2 a1 ATTCCATAC-AGAGGCTAATGGATACTGT CR-slcrcb-3 a1 ATTCCATAC-----GCTAATGGATACTGT CR-slcrcb-4 a1 ATTCCATAC-AGAGGCTAATGGATACTGT a2 ATTCCATAC--GAGGCTAATGGATACTGT

d
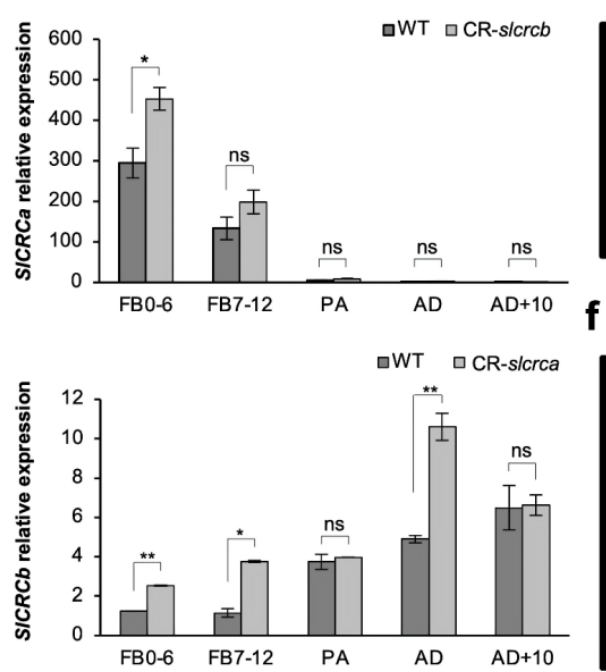

b

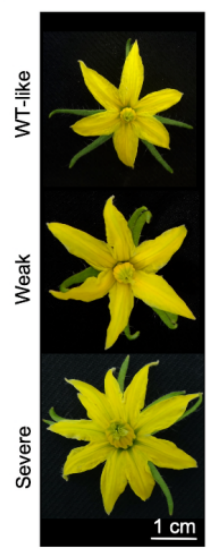

e

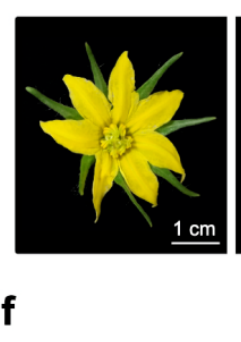

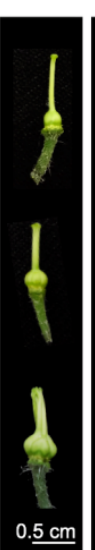

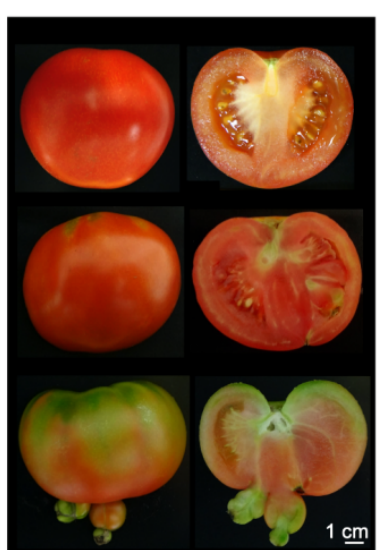

C

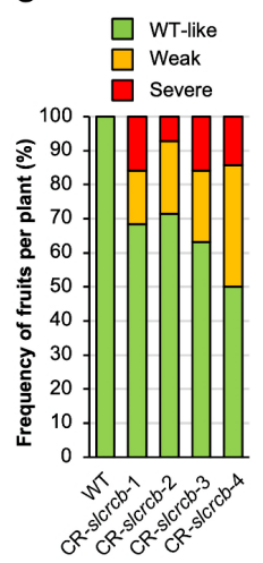

g
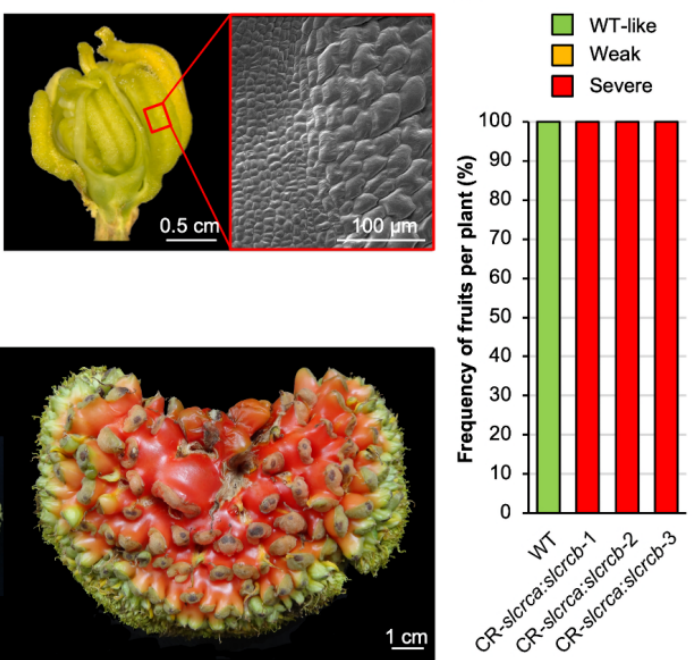

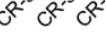

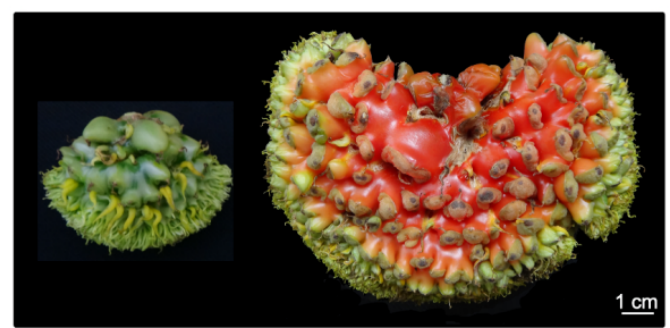

\section{Fig. 4 | Characterisation of CRISPR/Cas9-s/crcb (CR-s/crcb) and double}

425 mutant CR-s/crca:s/crcb lines. a, CR-s/crcb alleles identified by cloning and

426 sequencing PCR products from the SICRCb targeted region from four $\mathrm{T}_{0}$ CRISPR 427 plants. Black bold and underlined letters indicate protospacer-adjacent motif (PAM)

428 sequences, blue dashed lines show InDel mutations, blue letter and arrow indicate 429 an insertion sequence. b, Representative CR-s/crcb flowers, pistils and fruits 430 exhibiting WT-like, weak and severe phenotypes. c, Percentage of different types 431 of fruits harvested from wild-type (WT) and $T_{0}$ CR-s/crcb plants. d, Relative 
432 expression of SICRCa and SICRCb in CR-slcrcb and CR-slcrca lines, respectively, 433 at different floral developmental stages. FB0-6, floral buds from stages 0 to 6; FB7434 12, floral buds from stages 7 to 12; PA, flowers at preanthesis stage; AD, flowers at 435 anthesis stage; AD+10, flowers 10 days after anthesis stage. Data are means \pm 436 standard deviations (SD) of three biological and two technical replicates. A two437 tailed, two-sample Student's $t$ test was performed, and significant differences are 438 represented by asterisks: * $P<0.01$; **, $P<0.001$; ns, no statistically significant 439 differences. e,f, Representative flower, pistil and fruits developed by CR440 slcrca:slcrcb double mutants. e, Detail of the fourth floral whorl organs and 441 morphological features of their epidermal cells in a flower at anthesis stage. $\mathbf{f}$, 442 Immature green and mature red fruits. g, Percentage of different types of fruits 443 harvested from WT and To CR-s/crca:s/crcb plants.

Tomato CRC paralogues bind to the chromatin remodelling complex members repressing SIWUS expression

Floral determinacy requires the repression of the stem cell identity gene SIWUS ${ }^{6}$; therefore, we further investigated the function of tomato CRC paralogues in FM determination by examining the spatio-temporal expression of SIWUS in CR-slcrc lines by in situ hybridisation. In WT flowers, SIWUS expression in the organising centre was detected from early FM developmental stages (Chu et al., 2019), until stem cell activity was arrested in floral buds at stages 4-5, when the carpel 454 primordia started to emerge (Fig. 5a). From stage 6 onwards, SIWUS signal was 455 completely abolished in WT developing carpels. However, SIWUS transcripts were 456 detected, between the growing but still unfused carpel primordia, in both CR-s/crca 457 and CR-slcrcb floral buds at stage 6; and SIWUS expression was even observed at 458 later stages in a small group of cells in the placenta, where the initiation of new 459 carpel primordia probably occurs (Fig. 5a). As expected, an enlarged SIWUS 460 expression domain was observed in CR-slcrca:slcrcb flowers leading to an 461 increased FM size, which agrees with the severe indeterminacy found in double 462 mutant carpels (Fig. 5a). By way of conclusion, the extended expression of SIWUS 
463 correlates with the floral indeterminacy phenotype of CR-s/crc lines and suggests

464 that tomato CRC paralogues might participate in the regulation of SIWUS

465 transcription limiting FM activity and promoting floral determinacy.

466

467 Recent studies have proven that the complete termination of floral stem cell activity

468 in tomato is mediated by a chromatin remodelling complex, consisting of the SIIMA 469 (Solyc02g087970), SIKNU, (Solyc02g160370) SITPL (Solyc01g100050) and 470 SIHDA1 (Solyc09g091440) proteins, which enables the complete repression of 471 SIWUS (Bollier et al., 2018). The fact that SIWUS expression was mis-regulated in 472 CR-slcrca and CR-slcrcb indeterminate flowers prompted us to question whether 473 tomato CRC paralogues might be part of this regulatory pathway. Since SIKNU, 474 SIIMA, SITPL1 and SIHDA1 show nuclear localisation, we first evaluated SICRCa 475 and SICRCb subcellular localisation by transient expression of $\mathrm{N}$-terminal green 476 fluorescent protein (GFP)-tagged versions of SICRCa and SICRCb. A confocal 477 microscopy analysis revealed an exclusive nuclear localisation for both proteins 478 (Fig. 5b). Next, we conducted bimolecular fluorescence complementation (BiFC) 479 assays to evaluate whether tomato CRC paralogues might physically interact in 480 planta with the chromatin remodelling complex members including SIKNU, SIIMA, 481 SITPL1 and SIHDA1. Thus, BiFC interactions were tested by transiently co482 transforming with fusions of either the yellow fluorescent protein (YFP) C-terminal 483 region fused to SICRCa (SICRCa-cYFP) or SICRCb (SICRCb-cYFP), and fusions 484 of the YFP N-terminal region fused to either SIKNU (nYFP-SIKNU), SIIMA (nYFP485 SIIMA), SIHDA1 (nYFP-SIHDA1) or SITPL1 (nYFP-SITPL1). In addition, the 486 SICRCa-cYFP fusion was co-transfected with SICRCb fused to the YFP N-terminal 487 region (nYFP-SICRCb). As noted by the YFP fluorescent signal observed at the 488 nucleus of epidermal cells, SICRCa and SICRCb were able to physically interact 489 with each other and each of them with SIKNU, SIIMA, and SIHDA1, whereas 490 SICRCb was additionally found to bind to SITPL1 (Fig. 5c). A lack of interaction 491 between SICRCa and SITPL1 was also confirmed by BiFC experiments in the 492 opposite orientation (Supplementary Fig. 6). To corroborate the interactions 493 described above, they were furthermore tested by co-immunoprecipitation (ColP) 
494 studies. Thus, co-transfection was performed with either GFP-tagged SICRCa

495 (SICRCa $\left.{ }^{\text {GFP }}\right)$ or SICRCb $\left(\mathrm{SICRCb}^{\text {GFP }}\right)$ and the different hemagglutinin (HA)-tagged 496 members of the chromatin remodelling complex $\left(\mathrm{SIKUN}^{\mathrm{HA}}\right.$, SIIMA ${ }^{\mathrm{HA}}, \mathrm{SIHDA}^{\mathrm{HA}}$ or 497 SITPL1 $\left.{ }^{\mathrm{HA}}\right)$. Likewise, the SICRCb ${ }^{\mathrm{GFP}}$ was co-transfected with HA-tagged SICRCa $498\left(\mathrm{SICRCa}{ }^{\mathrm{HA}}\right)$. Total protein extracts were inmunoprecipitated with anti-GFP beads.

499 A Western blot analysis of the recovered fraction obtained using a specific antibody 500 to the HA epitope revealed that SICRCa ${ }^{\text {GFP }}$ copurified with SIKNU ${ }^{\mathrm{HA}}$, SIIMA ${ }^{\mathrm{HA}}$ and 501 SIHDA $1^{\mathrm{HA}}$, but not with SITPL $1^{\mathrm{HA}}$, as well as SICRCb ${ }^{\mathrm{GFP}}$ copurified with SIKNU ${ }^{\mathrm{HA}}$, 502 SIIMA $^{\mathrm{HA}}$, SIHDA $^{\mathrm{HA}}$, SITPL $1^{\mathrm{HA}}$ and SICRCa ${ }^{\mathrm{HA}}$ (Fig. 5d). Therefore, both BiFC and 503 ColP assays yielded concordant results, evidencing that SICRCa and SICRCb are 504 able to physically bind to the chromatin remodelling complex members, which in 505 turn repress SIWUS expression to promote FM determinacy. 
a

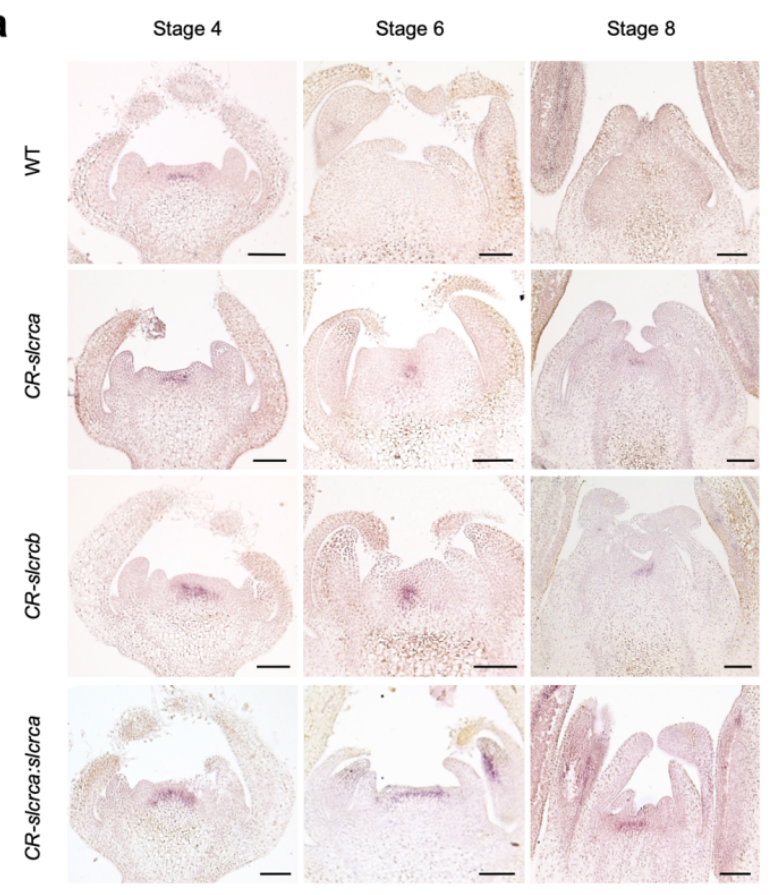

b

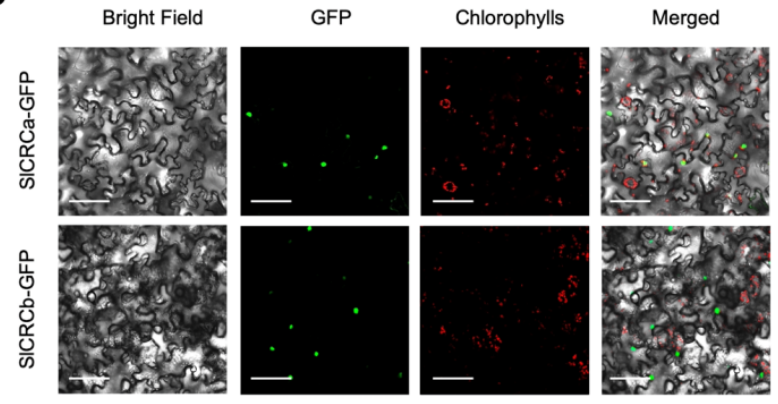

C

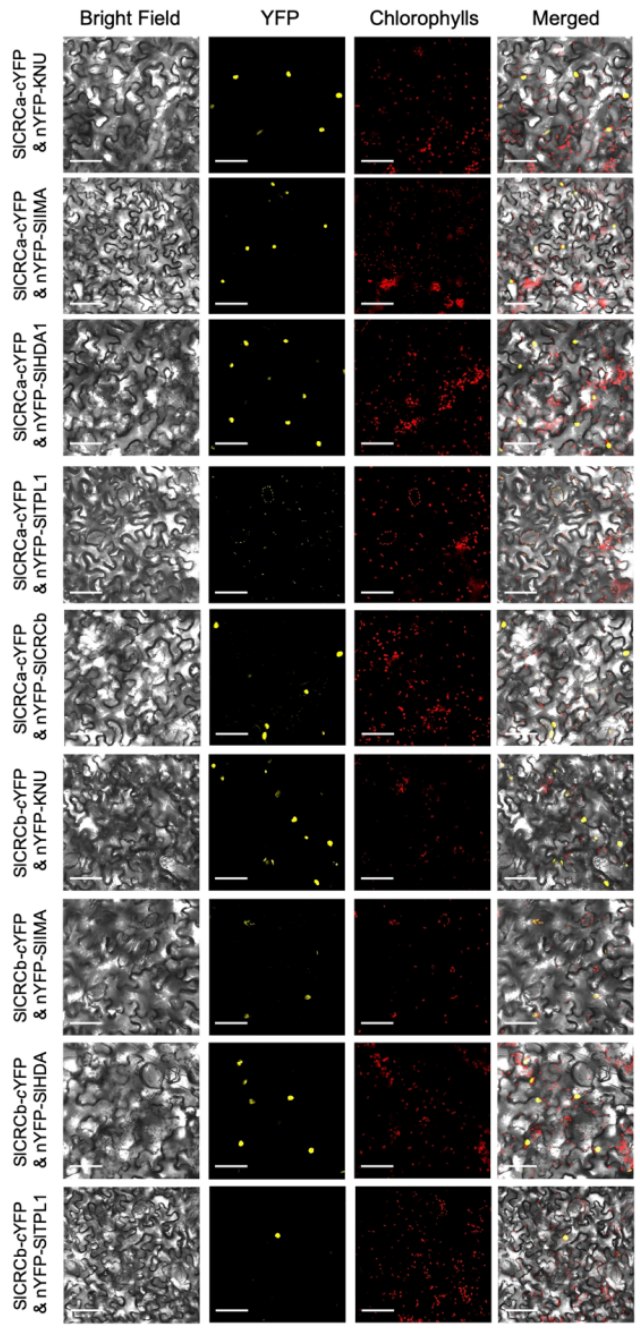

d

508 Fig. 5 | Tomato CRC paralogues interact with the chromatin remodelling 509 complex members repressing SIWUS expression. a, In situ mRNA hybridisation

510 of SIWUS in histological sections of wild-type (WT), CR-s/crca, CR-s/crcb and CR-

511 s/crca:s/crcb flowers at developmental stages 4, 6 and 8. b. Subcellular localisation

512 of SICRCa and SICRCb. The entire SICRCa and SICRCb coding sequences were

513 N-terminal fused to green fluorescent protein (GFP) and transiently expressed in

514 Nicotiana benthamiana leaves. c, Bimolecular fluorescence complementation 
515 confocal images showing in vivo interactions in $N$. benthamiana leaves between

516 either the yellow fluorescent protein (YFP) C-terminal region fused to SICRCa

517 (SICRCa-cYFP) or SICRCb (SICRCb-cYFP), and fusions of the YFP N-terminal

518 region fused to SIKNU (nYFP-SIKNU), SIIMA (nYFP-SIIMA), SIHDA1 (nYFP-

519 SIHDA1) or SITPL1 (nYFP-SITPL1). The SICRCa-cYFP fusion was also examined

520 with SICRCb fused to the YFP N-terminal region (nYFP-SICRCb). No YFP

521 fluorescence signal was observed in leaves infiltrated with a single construct and

522 the corresponding empty vector (Supplementary Fig. 6). d, Co-immunoprecipitation

523 studies of $N$. benthamiana leaves expressing either GFP-tagged SICRCa

$524\left(\mathrm{SICRCa}{ }^{\mathrm{GFP}}\right)$ or SICRCb $\left(\mathrm{SICRCb}^{\mathrm{GFP}}\right)$ and the different hemagglutinin (HA)-tagged

525 members of the chromatin remodelling complex $\left(\mathrm{SIKUN}^{\mathrm{HA}}\right.$, SIIMA ${ }^{\mathrm{HA}}, \mathrm{SIHDA}^{\mathrm{HA}}$ or

526 SITPL $\left.1^{\mathrm{HA}}\right)$. SICRCb ${ }^{\mathrm{GFP}}$ was also tested with HA-tagged SICRCa $\left(\mathrm{SICRCa}{ }^{\mathrm{HA}}\right)$. The

527 input total protein extracts were immunoprecipitated with anti-GFP beads, and the

528 unbound and recovered fractions (CoIP) were incubated with anti-GFP (Ab ${ }^{\text {GFP }}$ ) and

529 anti-HA $\left(\mathrm{Ab}^{\mathrm{HA}}\right)$ antibodies to detect precipitated and copurified proteins,

530 respectively. In a-c scale bars represent $100 \mu \mathrm{m}$.

532 Tomato CRC paralogues partially rescue the loss-of-function of the 533 Arabidopsis CRC gene

535 In Arabidopsis, mutations in CRC cause several phenotypic alterations affecting 536 the gynoecium which fails to fuse at the apex, making it wider and shorter than the 537 WT one (Alvarez and Smyth, 1999; Bowman and Smyth, 1999). As tomato CRC 538 paralogues seem to have partially redundant functions, we next questioned 539 whether they may be able to complement the phenotypic defects of Arabidopsis crc 540 mutants. Thus, the Arabidopsis crc-1 mutant, a strong hypomorphic allele in the 541 ecotype Landsberg erecta (Ler) genetic background, was genetically transformed 542 with either SICRCa or SICRCb coding sequences under the control of the 543 Arabidopsis CRC promoter ( $p C R C:: S I C R C a$ and $p C R C:: S I C R C b)$. For these 544 experiments, a 3860 bp fragment from upstream of the Arabidopsis CRC start 545 codon was used as promoter sequence since its functionality has been previously 
546 demonstrated (Fourquin et al., 2007). In comparison to WT Ler plants, crc-1 547 mutants exhibited unfused carpels and the abolition of nectary development, as 548 well as considerably shorter and wider siliques (Fig. 6). The pCRC::SICRCa and 549 pCRC::SICRCb transgenes were able to fully restore carpel fusion (Fig. 6c), and 550 slightly increased silique length (Fig. 6a,b). In agreement with the lack of nectaries 551 in tomato flowers, SICRCa and SICRCb did not rescue nectary development in crC5521 mutants (Fig. 6d). As a control, WT Ler plants were also transformed with either 553 pCRC::SICRCa and pCRC::SICRCb, which exhibited no significant differences with 554 respect to untransformed Ler plants (Supplementary Fig. 7). Overall, our results 555 denote that tomato CRC paralogues are capable of partially restoring a WT 556 phenotype when transformed into $\operatorname{crc}-1$ mutant plants.

a

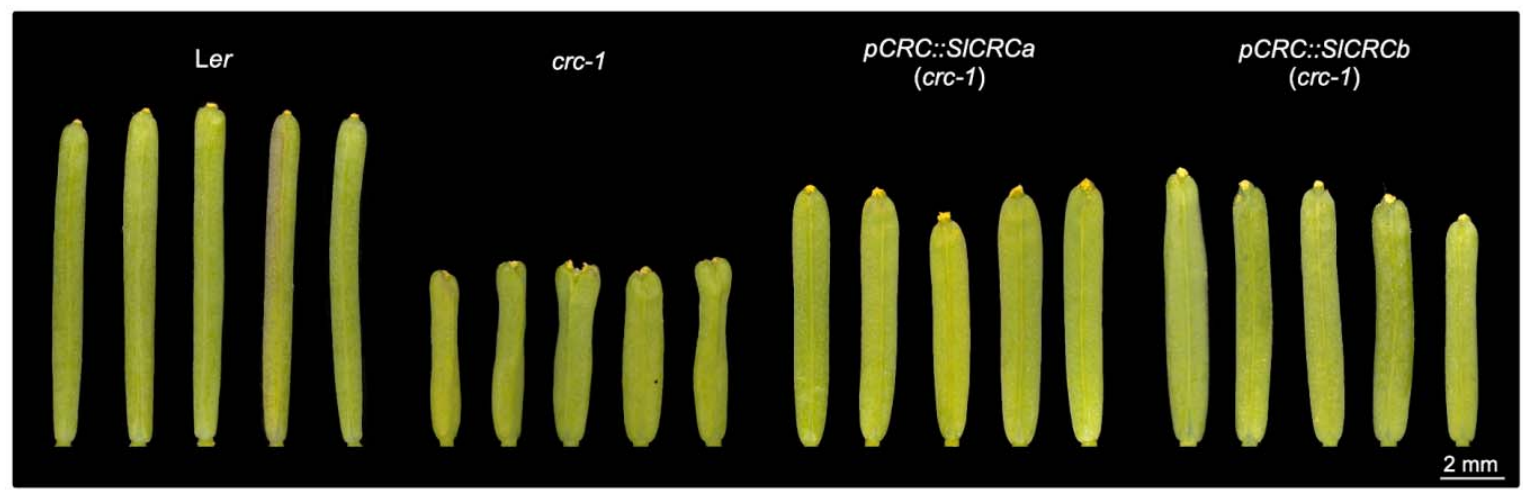

b

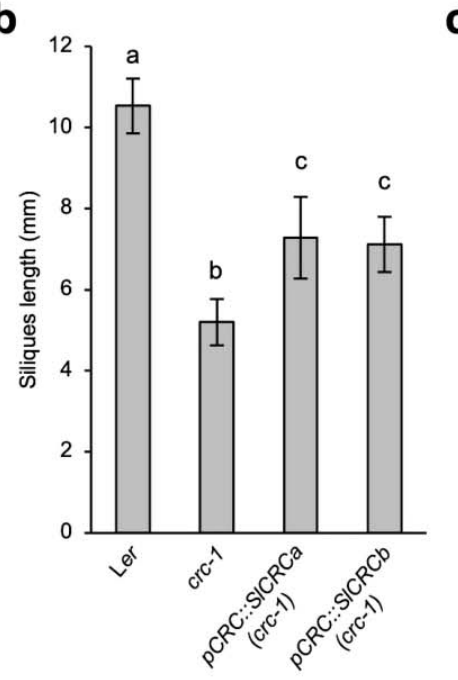

C

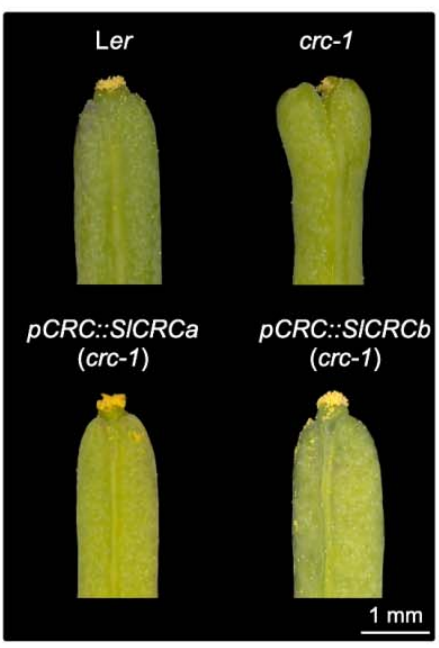

d

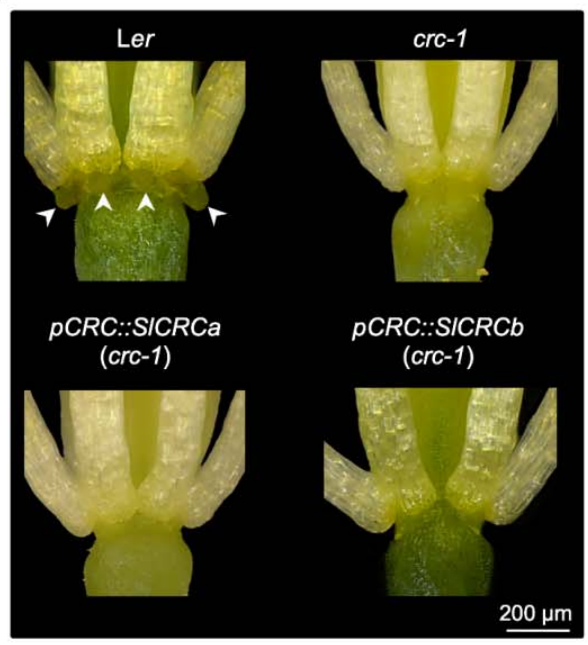

Fig. 6 | Complementation of the Arabidopsis crc-1 mutation by

560 transformation with tomato CRC paralogues. a-d, Fully elongated siliques (a), 
561 silique length (b), silique apices showing different degrees of carpel fusion (c), and 562 development of nectaries (arrows) at the base of the third floral whorl in Ler wild563 type, which are absent in crc-1 mutant and transgenic pCRC::SICRCa and 564 pCRC::SICRCb plants (d). In b pairwise comparisons of means using the least 565 significant difference test were performed. Values followed by the same letter (a, b, 566 or $\mathrm{c})$ are not statistically different $(P<0.05)$.

567

568 


\section{Discussion}

570

571 Tomato CRC paralogues safeguard floral determinacy by acting in a partially

572 redundant and compensatory manner

574 In the present study, we have addressed the functional characterization of tomato 575 paralogous CRC genes and examined their potential roles in FM determinacy and 576 carpel formation. Our results revealed that a $367 \mathrm{bp}$ insertion in the fourth intron of 577 the SICRCa gene, a homologue of the Arabidopsis CRC, causes the carpel-inside578 carpel phenotype observed in fig mutant plants. Loss-of-function analyses of 579 SICRCa, involving either knockdown (RNAi) or knockout (CRISPR/Cas9) 580 approaches, allowed for the generation of an allelic series at this locus which 581 resulted in analogous mutant phenotypes with incomplete penetrance and variable 582 expressivity. Indeed, variable floral and fruit phenotypes were observed even in the 583 same individual, ranging from indistinguishable from WT to severe developmental 584 defects. Consequently, these results denoted that any impairment of SICRCa 585 function would give rise to a loss of FM determinacy characterized by the 586 proliferation of additional carpels within the unfused primary carpels. Our results 587 also suggested that there is not a direct correlation between SICRCa mRNA levels 588 and the severity of the mutant phenotypes, which agrees with the hypothesis that 589 propose a non-linear gene dosage response for developmental regulators involved 590 in complex transcriptional regulatory networks (Birchler et al., 2016). The lack of 591 predictability between gene dosage and phenotypic alterations has been also 592 reported for other tomato meristem genes. Thus, through creating a series of cis593 regulatory alleles by genome editing, Rodríguez-Leal et al. (2017) demonstrated 594 that variations in fruit size are not predicted by changes in gene expression levels 595 of the CLAVATA-WUSCHEL signalling pathway.

597 Incomplete penetrance and variable expressivity of mutant phenotypes were also 598 observed among knockout mutant lines for SICRCb, whereas all flowers developed 599 by double mutant plants for SICRCa and SICRCb loci showed a severe 
600 indeterminate phenotype. Based on these results, we conclude that tomato CRC 601 paralogues operate as positive regulators of floral determinacy by acting in a 602 partially redundant manner to safeguard normal determination of FM and carpel 603 development. Indeed, the loss of carpel identity in CR-s/crca:s/crcb double mutant 604 plants supports the role of tomato CRC paralogues in establishing carpel identity 605 for proper completion of gynoecium and fruit developmental programs. 606 Furthermore, SICRCa was differentially up-regulated in tomato floral buds lacking 607 SICRCb function (CR-slcrcb plants), and vice versa, SICRCb expression 608 significantly increased in the absence of SICRCa activity (CR-slcrca plants). 609 Therefore, an active compensation mechanism of SICRCa and SICRCb functions 610 may participate in the regulation of FM determinacy. In support of this hypothesis, 611 transcriptional compensation has been recently described as a means to control 612 meristematic activity in tomato, where the CLV3/embryo-surrounding region (CLE) 613 ligand paralogues operate to buffer stem cell homeostasis (Rodriguez-Leal et al., 614 2019). Hence, the absence of SICRCa or SICRCb gene function would trigger an 615 active compensation mechanism involving the upregulation of SICRCb or SICRCa, 616 respectively, which help to buffer the severity of flower developmental alterations. 617 However, environmental or other genetic factors could lead to a partially 618 compensatory response influencing penetrance and expressivity of phenotypes 619 associated with single mutations at either SICRCa or SICRCb loci. Taken together, 620 our results indicate that tomato CRC paralogues operate as positive regulators of 621 FM determinacy by acting in a partially redundant and compensatory manner to 622 ensure normal floral development.

\section{Evolutionary conservation and divergence of CRC gene function in tomato}

626 Based on functional studies, the role of putative CRC orthologues in FM 627 determinacy and gynoecium development seems to have been conserved across 628 angiosperms, including monocots, basal dicots and eudicots (Bowman and Smyth, 629 1999; Yamaguchi et al., 2004; Lee et al., 2005a, 2005b; Fourquin et al., 2005, 630 2007, 2014; Nakayama et al., 2010; Bartholmes et al., 2012; Sun et al., 2013; 
631 Morel et al., 2018). Nevertheless, specialised functions of CRC-like genes have

632 been acquired after the evolutionary divergence of their respective plant lineages.

633 Thus, in monocot species such as rice, the CRC orthologue DROOPING LEAF

$634(D L)$ is also involved in carpel organ identity and plays an essential role in leaf 635 midrib formation, functions that are shared by CRC orthologues from other 636 monocot species (Yamaguchi et al., 2004; Ishikawa et al., 2009; Wang et al., 2009;

637 Nakayama et al., 2010; Strable et al., 2017). Likewise, novel functions in stigmatic 638 cavity formation and ovule initiation have been proposed for the paralogous CRC 639 genes PeDL1 and PeDL2 of the orchid Phalaenopsis equestri (Chen et al., 2021). 640 Within the Solanaceae family, the functional role of CRC paralogues has also been 641 addressed in petunia, where PhCRC1 and PhCRC2 genes, in addition to 642 regulating floral determinacy and carpel development, are required for nectary 643 development acting in a redundant manner to trigger its formation (Morel et al., 644 2018). PhCRC1 and PhCRC2 showed quasi-identical expression profiles, which 645 displayed an accumulation of their transcripts in carpel primordia, ovary walls, and 646 style and stigma (Morel et al., 2018), similar to the pattern observed for the tomato 647 CRC paralogues at the beginning of flower development. However, despite the fact 648 that tomato CRC paralogues play partially redundant roles to ensure normal floral 649 development, they were differentially expressed during flower ontogenesis. Thus, 650 SICRCa was mainly expressed at the first stage of the flower bud formation, 651 whereas SICRCb transcripts were detected from young floral buds to flowers at 10 652 days post anthesis, suggesting that their regulatory elements may have diverged. 653 In summary, although fine-tuning gene regulation events may have favoured gene 654 paralogue speciation, flower and fruit phenotypes of tomato plants lacking SICRCa 655 and SICRCb, together with gene expression analyses provide sufficient evidence 656 about the conserved function of these genes in regulating carpel development and 657 floral determinacy. Indeed, the heterologous expression of either SICRCa or 658 SICRCb genes in Arabidopsis crc-1 background was capable of partially restoring 659 a WT phenotype, supporting the evolutionary ancestral role of CRC-like genes in 660 promoting floral stem cell termination and carpel formation. However, tomato CRC 661 paralogues were not able to rescue the proper formation of nectaries. Previous 
662 studies have reported that CRC gene functions in nectary development appears to

663 be conserved in several core eudicot species, whereas CRC-like genes are not 664 required for nectary formation in basal angiosperms, which would support the 665 hypothesis that CRC function in nectary specification may be the consequence of 666 CRC neofunctionalization within diverse lineages of core eudicots (Lee et al., 667 2005b; Fourquin et al., 2005, 2014; Yamada et al., 2011).

669 In Arabidopsis and rice, CRC orthologue genes have also been found to act 670 antagonistically with class B genes in promoting carpel development (Alvarez and 671 Smyth, 1999; Bowman and Smyth, 1999; Yamaguchi et al., 2004). Here, we first 672 describe the CRC functional role in tomato, and we found that double mutant 673 plants for SICRCa and SICRCb loci develop flowers with stamen-like carpels 674 growing in a reiterating pattern inside the fourth whorl, which strongly hints that 675 tomato CRC paralogues seem to have common functions with the Arabidopsis and 676 rice counterparts as negative regulators of class B genes. Accordingly, a recent 677 research in the Solanaceae family member Physalis floridana, has revealed 678 regulatory and genetic interactions between B-class MADS-box and CRC genes in 679 a context-dependent manner during flower development (Gong et al., 2021).

Tomato CRC paralogues are part of the chromatin remodelling complex that repress SIWUS in floral meristems

684 A complex regulatory network involving signalling cascades, transcriptional 685 regulation, epigenetic mechanisms and hormonal control for FM determinacy has 686 been described in the model species Arabidopsis (Shang et al., 2019). Thus, the 687 function of CRC as regulator of floral determinacy has hitherto been limited to 688 modulate auxin homeostasis by both activating YUC4 and repressing TRN2 gene 689 expression (Yamaguchi et al., 2017, 2018). Our findings reveal a new role for 690 tomato CRC paralogues in balancing floral stem cell proliferation and 691 differentiation, as they are able to physically bind to the members of the chromatin 692 remodelling complex that drives the epigenetic regulation of SIWUS expression. In 
693 this epigenetic silencing mechanism, SIIMA acts as an adaptor protein engaging 694 SIKNU in a complex that involves SITPL and SIHDA1 leading to SIWUS repression 695 (Bollier et al., 2018). Based on the SIWUS expression profiles in flowers of single 696 and double mutants for SICRCa and SICRCb, as well as protein interaction data 697 hereby reported, it seems reasonable to propose a model by which SICRCa and 698 SICRCb would act in specifying floral determinacy by binding to the chromatin 699 remodelling complex that ensures the proper spatio-temporal repression of SIWUS 700 during flower development (Fig. 7). A future challenge will be to assess the role of 701 tomato CRC paralogues in regulating auxin homeostasis, as well as to determine 702 whether CRC interactions are conserved in angiosperm species. Further studies 703 on the degree of conservation or divergence in the molecular mechanism triggering 704 floral determinacy will provide valuable information for crop yield improvement, as 705 the number of carpels in a flower, and consequently the final number of locules 706 forming the mature fruit, plays a key role in regulating fruit size. In conclusion, this 707 research contributes to a better understanding of the genetic and molecular 708 mechanisms regulating FM determinacy, and hence influencing external fruit 709 quality, an important trait in tomato breeding. As it has been proven for the 710 CLAVATA-WUSCHEL signalling network (Rodríguez-Leal et al., 2017; Liu et al., 711 2021), the ever-increasing understanding of meristem regulatory pathways, and 712 their relevance in regulating crop yield, allow for new knowledge-driven breeding 713 strategies, which will in turn significantly contribute to the sustainability of 714 agriculture in the next decades. 


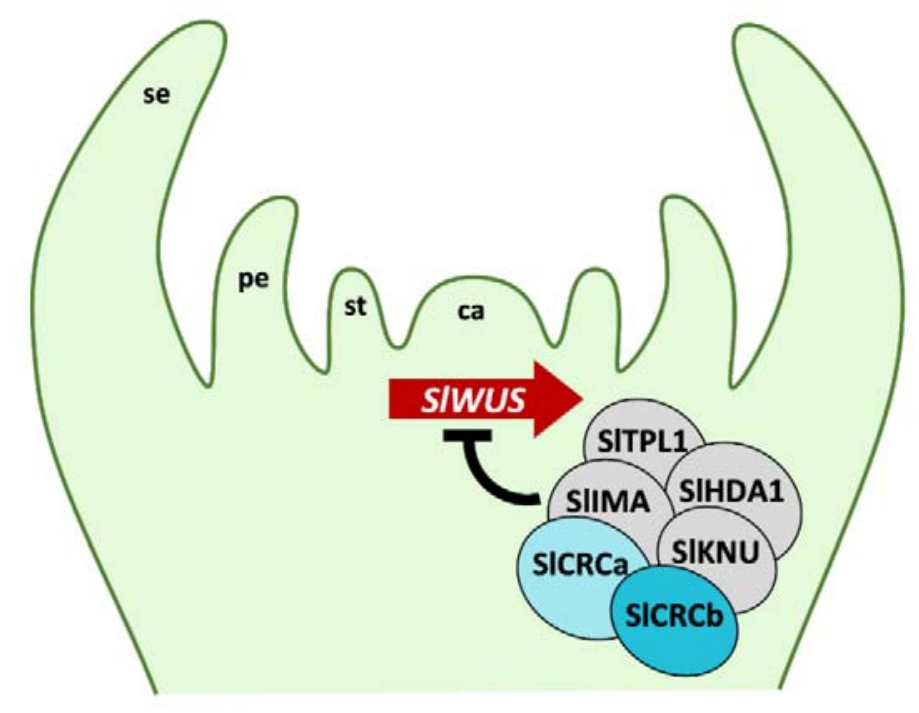

717 Fig. 7 | Proposed model for the function of tomato CRC paralogues in SIWUS

718 repression and floral meristem determinacy. Tomato CRC paralogues (SICRCa

719 and SICRCb), SIIMA and SIKNU interact with SIHDA1 and SITPL1 to form a

720 chromatin remodelling complex that represses SIWUS expression to terminate

721 floral stem cell activity once carpel primordia are initiated. se, sepal; pe, petal; st, 722 stamen; ca, carpel. 


\section{Methods}

726

\section{Plant material and growth conditions}

728

729 The fig mutant was identified from a T-DNA insertional mutant collection generated

730 in the genetic background cultivar P73 (Pérez-Martín et al., 2017). However,

731 molecular analysis showed that the fig mutation was not associated with a T-DNA

732 insertion (Supplementary Fig. 2). For mapping-by-sequencing, an $F_{2}$ mapping

733 population was generated by crossing a fig mutant to wild tomato Solanum

734 pimpinellifolium accession LA1589 and self-fertilizing the $\mathrm{F}_{1}$ plants. Tomato plants

735 were grown under greenhouse conditions with natural sunlight (13 to $14 \mathrm{~h}$

736 photoperiod). Temperatures ranged between $26-34^{\circ} \mathrm{C}$ and $20-25^{\circ} \mathrm{C}$ for daytime

737 and night-time periods, respectively. Standard management practices were used

738 including regular addition of fertilisers.

740 All Arabidopsis thaliana plants used for this study, including mutant and transgenic

741 plants, were in the ecotype Landsberg erecta (Ler) genetic background (kindly 742 provided by Prof. M.R. Ponce, Miguel Hernández University, Elche, Spain). Seeds

743 of the crabs claw-1 (crc-1; CS3814; N3814) mutant (Alvarez and Smyth, 1999)

744 were initially obtained from the Nottingham Arabidopsis Stock Center (NASC; 745 Nottingham, UK) and propagated at our laboratory for further analysis. Seeds were 746 surface sterilised for $8 \mathrm{~min}$ in $40 \%$ (v/v) sodium hypochlorite and 1\% (v/v) Triton X-

747100 with periodic agitation, rinsed at least three times with sterile distilled water 748 and sown in Petri dishes containing MS (Murashige and Skoog, 1962) growth 749 medium for germination. When required, kanamycin was added to the MS growth 750 medium at a final concentration of $50 \mu \mathrm{g} / \mathrm{ml}$. After stratification at $4^{\circ} \mathrm{C}$ for $24 \mathrm{~h}$ in 751 the dark, the plates were incubated in a growth chamber at $22^{\circ} \mathrm{C}$ with a $16 \mathrm{~h}$ light / $7528 \mathrm{~h}$ darkness cycle. For non-sterile conditions, plants were grown in pots at $25^{\circ} \mathrm{C}$ 753 and $60-70 \%$ relative humidity under constant illumination. 
757 The number of floral organs (sepals, petals, stamens, and carpels) was evaluated

758 in at least 60 flowers at anthesis stage (AD) showing WT-like, weak and severe 759 phenotypes. A minimum of 60 mature fruits were collected and used to calculate 760 the average fruit weight $(\mathrm{g})$, width $(\mathrm{mm})$, length $(\mathrm{mm})$ and number of locules per 761 phenotype. All values were expressed as the mean \pm standard deviation.

762 Significant differences were calculated by pairwise comparisons of means using 763 the least significant difference test.

765 For optical microscopy analysis, floral buds were harvested at different 766 developmental stages. Samples were fixed in FAE (10\% formaldehyde, 5\% acetic 767 acid, and 50\% absolute ethanol), dehydrated in ethanol series, embedded in 768 paraffin and cut using a Leica RM2035 microtome. Eight $\mu \mathrm{m}$ transversal sections 769 were stained in a 1\% Toluidine Blue solution and observed under a Leica DM6 B 770 microscope. Scanning electron microscopy analyses were performed as described 771 in Lozano et al. (1998). Floral bud samples were hand-dissected, fixed in FAEG 772 (10\% formaldehyde, 5\% acetic acid, 50\% absolute ethanol and $0.72 \%$ 773 glutaraldehyde) and dehydrated in ethanol series. Subsequently, tissues were 774 dried using a critical point dryer (Bal-Tec CPD 030) and gold coated in a Sputter 775 Coater (Bal Tec SCD005). Finally, samples were visualised using a Hitachi S$7763500 \mathrm{~N}$ scanning electron microscope at $10 \mathrm{kV}$.

Whole genome sequencing and allele frequency analysis

780 Mapping-by-sequencing was performed as described previously Yuste-Lisbona et 781 al. (2021). Briefly, two pools were formed using equal amounts of DNA from 25 782 mutant and 50 randomly selected wild-type plants. DNA quality control, Illumina 783 library construction and sequencing with $100 \mathrm{bp}$ paired-end reads on the Illumina 784 HiSeq2000 platform (Illumina, Inc., San Diego, CA) were carried out at the 785 Genome Center of the Max Planck Institute for Plant Breeding Research 786 (Cologne). The resulting short reads have been deposited in the Sequence Read 
787 Archive (SRA) database at the National Center for Biotechnology Information

788 (NCBI) under BioProject accession number PRJNA685617. Paired-end reads were

789 aligned to the tomato genome reference sequence version 4.0 (ITAG4.0) using

790 Bowtie2 version 2-2.0.0-b5 with default parameters (Langmead and Salzberg, 791 2012). Duplicated were removed with MarkDuplicates from the Picard tools 792 (http://broadinstitute.github.io/picard/). Variant calling analysis was carried out 793 using the HaplotypeCaller tool from GATK (DePristo et al., 2011). Next, BCFtools 794 from the SAMtools package (Li et al., 2009) was used to filter biallelic SNPs with a 795 minimum depth of 10 reads in each sample. After filtering, the allele frequency ratio 796 (i.e., non-reference allele counts / total allele counts) for biallelic variants was 797 calculated. Finally, to identify the chromosomal region where the fig mutation is 798 located, the average allele frequencies were plotted along each chromosome using 799 a custom script in the $R$ environment for statistical computing ( $R$ Development 800 Core Team, 2020) which uses a sliding window and step size of 1000 and 100 801 variants, respectively. Once the candidate genomic region to host the fig mutation 802 was determined, the identified variants encompassing this genomic region were 803 filtered based on the following criteria: i) variants that were heterozygous (0/1) and 804 homozygous (1/1) for the alternative allele in wild-type and mutant pools, 805 respectively; and ii) unique variants that were not previously reported in the 806 sequenced tomato genomes (Lin et al., 2014; The 100 Tomato Genome 807 Sequencing Consortium, 2014).

808

809 PCR genotyping of SICRCa locus

811 DNAzol® Reagent kit (Invitrogen Life Technologies, San Diego, CA, USA Genomic 812 DNA) was used to extract genomic DNA from young leaves of a segregating fig 813 line following the manufacturer's instructions. DNA quantification was carried out 814 using a NanoDrop 2000 spectrophotometer (Thermo Scientific, Wilmington, DE, 815 USA). SICRCa locus was genotyped using the SICRCa-Fg and SICRCa-Rg 816 primers which amplify a 332 bp fragment in wild-type homozygous plants, a 699 bp 
817 fragment in fig mutant plants and both of them in heterozygous ones. The

818 sequences of primers used are shown in Supplementary Table 2.

RNA isolation and Quantitative Real-Time PCR

822 Total RNA was extracted using TRIZOL (Invitrogen Life Technologies, San Diego,

$823 \mathrm{CA}$ ) according to the manufacturer's indications. RNA quantity was estimated using

824 a NanoDrop 2000 spectrophotometer (Thermo Scientific, Wilmington, DE, USA)

825 and quality was checked by gel electrophoresis. Contaminating DNA was removed

826 using the DNA-freeTM kit (Ambion, Austin, TX, USA) and $1 \mu \mathrm{g}$ of RNA was used

827 for cDNA synthesis with a ML-MLV reverse transcriptase (Invitrogen) with a

828 mixture of random hexamer and oligo-(dT) 18 primers. The qRT-PCR analysis was

829 carried out using three biological and two technical replicates. Specific primer pairs

830 were used in each qRT-PCR reaction with the SYBR Green PCR Master Mix kit

831 (Applied Biosystems, Foster City, CA, USA) on the 7300 Real-Time PCR System

832 (Applied Biosystems, Foster City, CA, USA). The housekeeping gene Ubiquitin3

833 (Solyc01g056940) was used as a control in all gene expression analyses.

834 Quantification of gene expression was performed using the $\Delta \Delta$ Ct calculation

835 method (Winer et al., 1999). Relative expression values were represented as the

836 mean \pm standard deviation. The sequences of primers used in this study are listed

837 in Supplementary Table 3.

\section{Generation of tomato transgenic lines}

841 To generate the RNA interference (RNAi) SICRCa construct, a 118 bp fragment of 842 the first exon of the Solyc01g0104120 cDNA was amplified using the SICRCa-Fi 843 and SICRCa-Ri primers and cloned in sense and antisense orientation into the 844 pKannibal vector (Wesley et al., 2001). The modified pKannibal vector was 845 digested with Notl and the resulting restriction fragment was cloned into the 846 pART27 binary vector (Gleave, 1992). CRISPR-Cas9 lines of SICRCa and SICRCb 847 genes were obtained following the protocol described by Vazquez-Vilar et al. 
848 (2016). Thus, Breaking-Cas web software (Oliveros et al., 2016) was used to 849 design the sgRNA target sequences within the coding region of each SICRC gene, 850 SICRCa (GTATCCAACAACTTCTTGCA) SICRCb

851 (GTATCCATTAGCCTCTTGTA). Each first-generation ( $\left.\mathrm{T}_{0}\right)$ transgenic plant was 852 genotyped with primers that cover the target recognition region of each sgRNA. 853 PCR products were cloned into the pGEM-T vector and at least 10 clones per PCR 854 product were sequenced. Finally, only biallelic or chimeric $\mathrm{T}_{0}$ plants for mutant 855 knockout alleles were phenotyped. The sequences of primers used in the 856 generation of RNAi and CRISPR/Cas9 constructs are shown in Supplementary 857 Table 2. Genetic transformation experiments were carried out as described in Ellul 858 et al. (2003). using Agrobacterium tumefaciens LBA4404 strain. The ploidy levels 859 of $\mathrm{T}_{0}$ transgenic plants were assessed by following as previously described by 860 Atarés et al. (2011). The CR-slcrca:s/crcb double mutant plants were generated 861 using standard crossing between single slcrcb and slcrca To CRISPR null mutants, 862 and were confirmed by genotyping from $\mathrm{F}_{1}$ progeny plants.

863

\section{In situ hybridisation analysis}

865

866 Tissue preparation, sectioning and transcript detection for in situ hybridisation

867 experiments were carried out as described in Lozano et al. (1998). Briefly, SICRCa 868 (Solyc01g0104120), SICRCb (Solyc05g012050) and SIWUS (Solyc11g071380) 869 probes were synthesised using cDNA as template by using the primers SICRCa870 Fz/SICRCa-Rz, SICRCb-Fz/SICRCb-Rz and SIWUS-Fz/SIWUS-Rz (sequences are 871 reported in Supplementary Table 3), respectively, and the resulting products were 872 cloned into the pGEM-T Easy vector (Promega, Madison, WI, USA). After plasmid 873 linearisation, the DIG RNA labelling mix (Roche Applied Science, Indianapolis, IN, 874 USA) and, depending on the orientation of the insert, T7 and SP6 polymerases 875 were used for in vitro transcription of antisense probes. As negative control, sense 876 RNA probes were synthesised and hybridised to sections of tomato floral buds. 
880 For expression analyses, three biological replicates per genotype were sequenced, 881 each with at least 30 floral buds at developmental stages 0-6. Total RNA was 882 isolated using TRIZOL (Invitrogen Life Technologies, San Diego, CA) following the 883 manufacturer's instructions. Contaminating DNA was removed using the DNA884 free $^{\mathrm{TM}}$ kit (Ambion, Austin, TX, USA). Libraries were prepared according to the 885 Illumina TruSeq RNA protocol and sequenced with paired-end $150 \mathrm{bp}$ on the 886 Illumina HiSeq2000 platform (Illumina, Inc., San Diego, CA, USA). The resulting 887 short reads were deposited in the SRA database at the NCBI under BioProject 888 accession number PRJNA686085. The sequence reads were aligned to the tomato 889 genome reference sequence version 4.0 (ITAG4.0) using Tophat v2.0.6 (Kin et al., 890 2013) with the following parameters: --max-insertion-length 12 --max-deletion891 length 12 -g 1 --read-gap-length 12 --read-edit-dist 20 --read-mismatches 12 --no892 coverage-search --read-realign-edit-dist 0 --segment-mismatches 3 --splice893 mismatches 1 . The raw number of reads per transcript was counted using the 894 Bioconductor packages GenomicFeatures and GenomicAlignments (Lawrence et 895 al., 2013). Differentially expressed genes were determined using the Wald test in 896 the DEseq2 package (Love et al., 2014) with the Benjamini-Hochberg multiple 897 testing correction (Benjamini and Hochberg, 1995) for false discovery rate (FDR). 898 Genes with an FDR adjusted P-value of less than 0.05 were defined as 899 significantly up- or down- regulated. Gene Ontology (GO) term enrichment analysis 900 of the significantly differentially expressed genes was performed using agriGO v2.0 901 software (Tian et al., 2017) and determined by FDR $<0.05$ with the Fisher 902 statistical test and the Bonferroni multi-test adjustment.

Molecular complementation of the Arabidopsis crc-1 mutant

906 The Arabidopsis crc-1 mutant was complemented with two different constructs, 907 each carrying a 3860 bp fragment from upstream of the Arabidopsis CRC start 908 codon representing its promoter region $(p C R C)$, fused to the coding sequences of 909 either the tomato SICRCa (pCRC::SICRCa) or SICRCb (pCRC::SICRCb) genes. 
910 The primers used for PCR amplification of $p C R C$ and genes coding regions are

911 shown in Supplementary Table 4. PCR products were purified and cloned into the

912 pGEMT vector (Promega, Madison, WI, USA). pCRC and each gene coding

913 fragments were linked by a double digestion with $X$ hol and a specific restriction

914 enzyme which cuts within the pGEM-T polylinker (Aatll or Sacl depending on the

$915 p C R C$ and coding sequences orientation in the pGEM-T) and subsequent ligation

916 conducted by T4 DNA ligase. The complete sequences of the $p C R C$ fused to the

917 corresponding gene coding sequence were obtained by amplifying with pCRC-

918 Fac/SICRCa-Rac or pCRC-Fac/SICRCb-Rac primers (Supplementary Table 4),

919 then cloned into the pENTR/D-TOPO vector (Invitrogen Life Technologies, San

920 Diego, CA, USA), and finally subcloned into the Gateway binary vector pGWB401

921 (Nakagawa et al., 2007). All final plasmids were verified by Sanger sequencing and

922 then transformed into $A$. tumefaciens C58C1 strain. The control WT Ler and the

923 mutant $c r c-1$ plants were transformed by the floral dip method described by Clough

924 and Bent (1998). $T_{1}$ transgenic plants were selected on plates supplemented with

$92550 \mu \mathrm{g} / \mathrm{mL}$ kanamycin as previously described in Harrison et al. (2006). At least 30

926 flowers and siliques from Ler, crc-1 and $\mathrm{T}_{1}$ transgenic plants, resulting from the

927 transformation of Ler and crc-1 plants with either pCRC::SICRCa or

928 pCRC::SICRCb constructs, were evaluated on a Leica DMS1000 digital

929 microscope.

931 Sequence alignment and microsynteny analysis

933 The amino acid sequences of SICRCa (XP_004228849), SICRCb (XP_004239032)

934 and CRC (NP_177078) were downloaded from the GenBank database

935 (http://www.ncbi.nlm.nih.gov) and pairwise aligned using BLASTp

936 (https://blast.ncbi.nlm.nih.gov/Blast.cgi). The study of microsynteny conservation

937 between the genomic regions harbouring the Arabidopsis CRC gene and either the

938 tomato SICRCa or SICRCb genes was carried out with the GEvo tool available in

939 the CoGe server (https://genomevolution.org/coge/GEvo.pl). Thus, pairwise

940 genomic alignments were performed between the genomic region comprising 
$94110,000 \mathrm{nt}$ upstream and downstream of the Arabidopsis CRC gene (chromosome 1

942 positions: 25,997,465-26,019,059; TAIR v10.02), and either 30,000 nt of the

943 genomic region flanking the tomato SICRCa (chromosome 1 positions: 5,022,049-

$9445,084,644 ;$ ITAG4.0) or SICRCb (chromosome 5 positions: 5,323,844-5,385,856;

945 ITAG4.0) genes.

947 Subcellular localisation and bimolecular fluorescence complementation

948 assays

950 SICRCa and SICRCb subcellular localisations were assessed by fusing each 951 protein to the green fluorescent protein (GFP). Thus, full-length open reading frame 952 sequences coding for SICRCa and SICRCb proteins were cloned into the 953 pENTR/D-TOPO vector (Invitrogen Life Technologies, San Diego, CA, USA) and 954 recombined into the Gateway binary vector pGWB6 (Nakagawa et al., 2007) to 955 integrate GFP at the N-terminus of the proteins of interest. To test for bimolecular 956 fluorescence complementation (BiFC)-based protein-protein interaction, full-length 957 open reading frame sequences of SICRCa, SICRCb, SIKNU, SIIMA, SIHDA1 and 958 SITPL1 proteins were cloned into the pENTR/D-TOPO vector and subloned into 959 the Gateway binary vectors containing the $\mathrm{N}$ - or C-terminal fragments of the yellow 960 fluorescent protein (pYFN43 and pYFC43 vectors, respectively). Constructs were 961 transformed in A. tumefaciens GV3101 strain and infiltrated into Nicothiana 962 benthamiana leaves from 2-3-week-old plants. Plants were kept in long-day (16 h 963 light/8 h dark) conditions at $22^{\circ} \mathrm{C}$. Samples were observed 3 days post infiltration 964 using a Nikon ECLIPSE Ti confocal microscope equipped with Nikon C2 Si lasers.

965 The sequences of primers used for subcellular localisation and BiFC assays are 966 shown in Supplementary Table 5.

\section{Co-immunoprecipitation assays}

970 For co-immunoprecipitation assays, N. benthamiana leaves were transiently co-

971 transfected with $A$. tumefaciens GV3101 strain cultures expressing either GFP- 
972 tagged SICRCa (SICRCa $\left.{ }^{\text {GFP }}\right)$ or SICRCb (SICRCb $\left.{ }^{\text {GFP }}\right)$ and the different

973 hemagglutinin (HA)-tagged members of the chromatin remodelling complex $974\left(\mathrm{SIKUN}{ }^{\mathrm{HA}}\right.$, SIIMA ${ }^{\mathrm{HA}}$, SIHDA $1^{\mathrm{HA}}$ or SITPL1 $\left.{ }^{\mathrm{HA}}\right)$. Likewise, the SICRCb ${ }^{\mathrm{GFP}}$ was co975 transfected with HA-tagged SICRCa (SICRCa ${ }^{\mathrm{HA}}$ ). For this purpose, pENTR/D-

976 TOPO vectors containing full-open reading frame sequences of the recombinant 977 fusion proteins of interest were recombined into the Gateway binary vectors 978 pGWB6 and pGWB15 (Nakagawa et al., 2007), containing GFP or HA tags, 979 respectively. These vectors were co-infiltrated in N. benthamiana leaves from 2-3980 week-old plants. Plants were kept under long-day (16 h light/8 h dark) conditions at $98122^{\circ} \mathrm{C}$. Subsequent protein extraction was performed from leaves harvested 2 days 982 after infiltration. Plant material was ground in liquid nitrogen and homogenised in 983 protein extraction buffer $(25 \mathrm{mM}$ Tris- $\mathrm{HCl}, \mathrm{pH} 7.4,75 \mathrm{mM} \mathrm{NaCl}, 0.5 \%$ Nonidet P984 40, 0.05\% sodium deoxycholate, $10 \mathrm{mM} \beta$-mercaptoethanol, $1 \mathrm{mM} \mathrm{PMSF}$, and 985 cOmplete-EDTA free protease inhibitor cocktail (Roche Applied Science, 986 Indianapolis, IN, USA). Protein extracts were centrifuged twice at 14,000 g for 10 987 min at $4^{\circ} \mathrm{C}$. After cell lysis, GFP-tagged proteins were magnetically labelled and 988 subsequent isolated using MMACS Isolation Kit (Miltenyi Biotec S.L., Bergisch 989 Gladbach, Germany) following manufacturer's instructions. The resulting samples 990 were analysed by SDS-PAGE and immunoblotted using anti-GFP-HPR (Miltenyi 991 Biotec, Bergisch Gladbach, Germany) and anti-HA-peroxidase (Roche Applied 992 Science, Basel, Switzerland) antibodies.

\section{Accession numbers}

995 Tomato sequence data from this article can be found at the SOL Genomics 996 Network (SGN; http://sgn.cornell.edu) under accession numbers Solyc01g010240 997 (SICRCa), Solyc05g012050 (SICRCb), $\quad$ Solyc11g071380 (SIWUS), 998 Solyc02g087970 (SIIMA), Solyc02g160370 (SIKNU), Solyc01g100050 (SITPL), 999 and Solyc09g091440 (SIHDA1). Sequence data from Arabidopsis can be found at 1000 the Arabidopsis Information Resource (TAIR; https://www.arabidopsis.org/) under 1001 the accession number At1g69180 (CRC). The DNA-seq and RNA-seq data from 1002 this article can be found at the Sequence Read Archive (SRA; 
1003 https://www.ncbi.nlm.nih.gov/sra/) under BioProject accession numbers 1004 PRJNA685617 and PRJNA686085, respectively.

1005

1006

Acknowledgments

1007

1008 The authors thank Cristina Ferrándiz for critical review of the manuscript. This work

1009 was supported by the PID2019-110833RB-C31 and PID2019-110833RB-C32

1010 grants from the Spanish Ministry of Science and Innovation (MICI/AEI/FEDER, UE)

1011 and the BRESOV (Breeding for Resilient, Efficient and Sustainable Organic

1012 Vegetable production) project funding by the Research and Innovation Programme

1013 of the European Union Horizon 2020 (grant agreement No. 774244). The authors

1014 also thank research facilities provided by the Campus de Excelencia Internacional

1015 Agroalimentario (CeiA3).

1016

1017 Author contributions

1018

1019 L.C. performed most experimental procedures, prepared figures and co-wrote the 1020 manuscript. E.G., B.P., B.G.-S., A.O.-A. and R.M.-P. participated in the 1021 experimental research. T.A., J.C., V.M., F.J.Y.-L., and R.L. contributed materials 1022 and genetic resources, and analysed data. F.J.Y.-L., and R.L. conceived and 1023 supervised research and co-wrote the paper.

\section{Conflict of interest statement}

1026 The authors declare that they have no conflict of interests. 


\section{Parsed Citations}

Aichinger E, Kornet N, Friedrich T, Laux T (2012) Plant stem cell niches. Annu Rev Plant Biol 63: 615-636

Google Scholar: Author Only Title Only Author and Title

Alvarez J, Smyth DR (1999) CRABS CLAW and SPATULA, two Arabidopsis genes that control carpel development in parallel with AGAMOUS. Development 126: 2377-2386

Google Scholar: Author Only Title Only Author and Title

Atarés A, Moyano E, Morales B, Schleicher P, García-Abellán JO, Antón T, García-Sogo B, Perez-Martin F, Lozano R, Flores FB, et al. (2011) An insertional mutagenesis programme with an enhancer trap for the identification and tagging of genes involved in abiotic stress tolerance in the tomato wild-related species Solanum pennellii. Plant Cell Rep 30: 1865-1879

Google Scholar: Author Only Title Only Author and Title

Bartholmes C, Hidalgo O, Gleissberg S (2012) Evolution of the YABBY gene family with emphasis on the basal eudicot Eschscholzia californica (Papaveraceae): YABBY genes in Eschscholzia californica. Plant Biol 14: 11-23

Google Scholar: Author Only Title Only Author and Title

Benjamini Y, Hochberg Y (1995) Controlling the false discovery rate: a practical and powerful approach to multiple testing. J R Stat Soc B 57: 289-300

Google Scholar: Author Only Title Only Author and Title

Birchler JA, Johnson AF, Veitia RA(2016) Kinetics genetics: incorporating the concept of genomic balance into an understanding of quantitative traits. Plant Sci 245: 128-134

Google Scholar: Author Only Title Only Author and Title

Bollier N, Sicard A, Leblond J, Latrasse D, Gonzalez N, Gévaudant F, Benhamed M, Raynaud C, Lenhard M, Chevalier C, et al. (2018) At-MINI ZNC FINGER2 and SI-INHIBITOR OF MERISTEM ACTIVTY, a conserved missing link in the regulation of floral meristem termination in Arabidopsis and tomato. Plant Cell 30: 83-100

Google Scholar: Author Only Title Only Author and Title

Bowman JL, Smyth DR (1999) CRABS CLAW, a gene that regulates carpel and nectary development in Arabidopsis, encodes a novel protein with zinc finger and helix-loop-helix domains. Development 126: 2387-2396

Google Scholar: Author Only Title Only Author and Title

Brukhin V, Hernould M, Gonzalez N, Chevalier C, Mouras A(2003) Flower development schedule in tomato Lycopersicon esculentum cv. sweet cherry. Sex Plant Reprod 15: 311-320

Google Scholar: Author Only Title Only Author and Title

Chen YY, Hsiao YY, Li Cl, Yeh CM, Mitsuda N, Yang HX, Chiu CC, Chang SB, Liu ZJ, Tsai WC (2021) The ancestral duplicated DL/CRC orthologs, PeDL1 and PeDL2, function in orchid reproductive organ innovation. J Exp Bot 72: 5442-5461

Google Scholar: Author Only Title Only Author and Title

Chu Y, Jang J, Huang Z, van der Knaap E (2019) Tomato locule number and fruit size controlled by natural alleles of IC and fas. Plant Direct 3: e00142

Google Scholar: Author Only Title Only Author and Title

Clough SJ, Bent AF (1998) Floral dip: a simplified method for Agrobacterium-mediated transformation of Arabidopsis thaliana. Plant $\mathrm{J}$ 16: 735-743

Google Scholar: Author Only Title Only Author and Title

DePristo MA, Banks E, Poplin R, Garimella KV, Maguire JR, Hartl C, Philippakis AA, del Angel G, Rivas MA, Hanna M, et al. (2011) A framework for variation discovery and genotyping using next-generation DNA sequencing data. Nat Genet 43: 491-498

Google Scholar: Author Only Title Only Author and Title

Ditta G, Pinyopich A, Robles P, Pelaz S, Yanofsky MF (2004) The SEP4 gene of Arabidopsis thaliana functions in floral organ and meristem identity. Curr Biol 14: 1935-1940

Google Scholar: Author Only Title Only Author and Title

Ellul P, Garcia-Sogo B, Pineda B, Ríos G, Roig LA, Moreno V (2003) The ploidy level of transgenic plants in Agrobacterium-mediated transformation of tomato cotyledons (Lycopersicon esculentum Mill.) is genotype and procedure dependent. Theor Appl Genet 106: 231-238

Google Scholar: Author Only Title Only Author and Title

Fourquin C, Primo A, Martínez-Fernández I, Huet-Trujillo E, Ferrándiz C (2014) The CRC orthologue from Pisum sativum shows conserved functions in carpel morphogenesis and vascular development. Ann Bot 114: 1535-1544

Google Scholar: Author Only Title Only Author and Title

Fourquin C, Vinauger-Douard M, Chambrier P, Berne-Dedieu A, Scutt CP (2007) Functional Conservation between CRABS CLAW orthologues from widely diverged angiosperms. Ann Bot 100: 651-657

Google Scholar: Author Only Title Only Author and Title

Fourquin C, Vinauger-Douard M, Fogliani B, Dumas C, Scutt CP (2005) Evidence that CRABS CLAW and TOUSLED have conserved 
their roles in carpel development since the ancestor of the extant angiosperms. Proc Natl Acad Sci U S A 102: 4649-4654

Google Scholar: Author Only Title Only Author and Title

Gleave AP (1992) Aversatile binary vector system with a T-DNA organisational structure conducive to efficient integration of cloned DNA into the plant genome. Plant Mol Biol 20: 1203-1207

Google Scholar: Author Only Title Only Author and Title

Gong P, Song C, Liu H, Li P, Zhang M, Zhang J, Zhang S, He C (2021). PFCRC mediates neofunctionalization of Physalis GLOBOSA genes in carpel development. J Exp Bot erab309. doi: 10.1093/jxb/erab309

Google Scholar: Author Only Title Only Author and Title

Gremski K, Ditta G, Yanofsky MF (2007) The HECATE genes regulate female reproductive tract development in Arabidopsis thaliana. Development 134: 3593-3601

Google Scholar: Author Only Title Only Author and Title

Guo L, Cao X, Liu Y, Li J, Li Y, Li D, Zhang K, Gao C, Dong A, Liu X (2018) Achromatin loop represses WUSCHEL expression in Arabidopsis. Plant J 94: 1083-1097

Google Scholar: Author Only Title Only Author and Title

Harrison SJ, Mott EK, Parsley K, Aspinall S, Gray JC, Cottage A(2006) A rapid and robust method of identifying transformed Arabidopsis thaliana seedlings following floral dip transformation. Plant Methods 2: 19

Google Scholar: Author Only Title Only Author and Title

Ishikawa M, Ohmori Y, Tanaka W, Hirabayashi C, Murai K, Ogihara Y, Yamaguchi T, Hirano HY (2009) The spatial expression patterns of DROOPING LEAF orthologs suggest a conserved function in grasses. Genes Genet Syst 84: 137-146

Google Scholar: Author Only Title Only Author and Title

Kagale S, Rozwadowski K (2011) EAR motif-mediated transcriptional repression in plants: An underlying mechanism for epigenetic regulation of gene expression. Epigenetics 6: 141-146

Google Scholar: Author Only Title Only Author and Title

Kim D, Pertea G, Trapnell C, Pimentel H, Kelley R, Salzberg SL (2013) TopHat2: accurate alignment of transcriptomes in the presence of insertions, deletions and gene fusions. Genome Biol 14: R36

Google Scholar: Author Only Title Only Author and Title

Krizek BA, Fletcher JC (2005) Molecular mechanisms of flower development: an armchair guide. Nat Rev Genet 6: 688-698

Google Scholar: Author Only Title Only Author and Title

Krogan NT, Hogan K, Long JA(2012) APETALA2 negatively regulates multiple floral organ identity genes in Arabidopsis by recruiting the co-repressor TOPLESS and the histone deacetylase HDA19. Development 139: 4180-4190

Google Scholar: Author Only Title Only Author and Title

Kwon CS, Chen C, Wagner D (2005) WUSCHEL is a primary target for transcriptional regulation by SPLAYED in dynamic control of stem cell fate in Arabidopsis. Genes Dev 19: 992-1003

Google Scholar: Author Only Title Only Author and Title

Langmead B, Salzberg SL (2012) Fast gapped-read alignment with Bowtie 2. Nat. Methods 9: 357-359

Google Scholar: Author Only Title Only Author and Title

Laux T, Mayer KF, Berger J, Jürgens G (1996) The WUSCHEL gene is required for shoot and floral meristem integrity in Arabidopsis.

Development 122: 87-96

Google Scholar: Author Only Title Only Author and Title

Lawrence M, Huber W, Pagès H, Aboyoun P, Carlson M, Gentleman R, Morgan MT, Carey VJ (2013) Software for computing and annotating genomic ranges. PLoS Comput Biol 9: e1003118

Google Scholar: Author Only Title Only Author and Title

Lee JY, Baum SF, Avarez J, Patel A, Chitwood DH, Bowman JL (2005) Activation of CRABS CLAW in the nectaries and carpels of Arabidopsis. Plant Cell 17: 25-36

Google Scholar: Author Only Title Only Author and Title

Lee JY, Baum SF, Oh SH, Jiang CZ, Chen JC, Bowman JL (2005) Recruitment of CRABS CLAW to promote nectary development within the eudicot clade. Development 132: 5021-5032

Google Scholar: Author Only Title Only Author and Title

Li H, Handsaker B, Wysoker A, Fennell T, Ruan J, Homer N, Marth G, Abecasis G, Durbin R, 1000 Genome Project Data Processing Subgroup (2009) The Sequence Alignment/Map format and SAMtools. Bioinformatics 25: 2078-2079

Google Scholar: Author Only Title Only Author and Title

Lin T, Zhu G, Zhang J, Xu X, Yu Q, Zheng Z, Zhang Z, Lun Y, Li S, Wang X, et al. (2014) Genomic analyses provide insights into the history of tomato breeding. Nat Genet 46: 1220-1226

Google Scholar: Author Only Title Only Author and Title

Liu L, Gallagher J, Arevalo ED, Chen R, Skopelitis T, Wu Q, Bartlett M, Jackson D (2021) Enhancing grain-yield-related traits by 
CRISPR-Cas9 promoter editing of maize CLE genes. Nat Plants 7: 287-294

Google Scholar: Author Only Title Only Author and Title

Liu X, Kim YJ, Müller R, Yumul RE, Liu C, Pan Y, Cao X, Goodrich J, Chen X (2011) AGAMOUS terminates floral stem cell maintenance in Arabidopsis by directly repressing WUSCHEL through recruitment of Polycomb Group proteins. Plant Cell 23: $3654-3670$

Google Scholar: Author Only Title Only Author and Title

Love MI, Huber W, Anders S (2014) Moderated estimation of fold change and dispersion for RNAseq data with DESeq2. Genome Biol 15: 550

Google Scholar: Author Only Title Only Author and Title

Lozano R, Angosto T, Gomez P, Payan C, Capel J, Huijser P, Salinas J, Martinez-Zapater JM. (1998) Tomato flower abnormalities induced by low temperatures are associated with changes of expression of MADS-Box genes. Plant Physiol 117: 91-100

Google Scholar: Author Only Title Only Author and Title

Mayer KF, Schoof H, Haecker A, Lenhard M, Jürgens G, Laux T (1998) Role of WUSCHEL in regulating stem cell fate in the Arabidopsis shoot meristem Cell 95: 805-815

Google Scholar: Author Only Title Only Author and Title

Morel P, Heijmans K, Ament K, Chopy M, Trehin C, Chambrier P, Rodrigues Bento S, Bimbo A, Vandenbussche M (2018) The floral Clineage genes trigger nectary development in Petunia and Arabidopsis. Plant Cell 30: 2020-2037

Google Scholar: Author Only Title Only Author and Title

Muños S, Ranc N, Botton E, Bérard A, Rolland S, Duffé P, Carretero Y, Le Paslier MC, Delalande C, Bouzayen M, et al. (2011) Increase in tomato locule number is controlled by two single-nucleotide polymorphisms located near WUSCHEL. Plant Physiol 156: 2244-2254

Google Scholar: Author Only Title Only Author and Title

Murashige T, Skoog F (1962) Arevised medium for rapid growth and bio assays with tobacco tissue cultures. Physiol Plant 15: 473-497

Google Scholar: Author Only Title Only Author and Title

Nakagawa T, Kurose T, Hino T, Tanaka K, Kawamukai M, Niwa Y, Toyooka K, Matsuoka K, Jinbo T, Kimura T (2007) Development of series of gateway binary vectors, pGWBs, for realizing efficient construction of fusion genes for plant transformation. $J$ Biosci Bioeng 104: 34-41

Google Scholar: Author Only Title Only Author and Title

Nakayama H, Yamaguchi T, Tsukaya H (2010) Expression patterns of AaDL, a CRABS CLAW ortholog in Asparagus asparagoides (Asparagaceae), demonstrate a stepwise evolution of CRC / DL subfamily of YABBY genes. Am J Bot 97: 591-600

Google Scholar: Author Only Title Only Author and Title

Oliveros JC, Franch M, Tabas-Madrid D, San-León D, Montoliu L, Cubas P, Pazos F (2016) Breaking-Cas-interactive design of guide RNAs for CRISPR-Cas experiments for ENSEMBL genomes. Nucleic Acids Res 44: W267-W271

Google Scholar: Author Only Title Only Author and Title

Payne T, Johnson SD, Koltunow AM (2004) KNUCKLES (KNU) encodes a C2H2 zinc-finger protein that regulates development of basal pattern elements of the Arabidopsis gynoecium. Development 131: 3737-3749

Google Scholar: Author Only Title Only Author and Title

Pérez-Martín F, Yuste-Lisbona FJ, Pineda B, Angarita-Díaz MP, García-Sogo B, Antón T, Sánchez S, Giménez E, Atarés A, FernándezLozano A et al. (2017) A collection of enhancer trap insertional mutants for functional genomics in tomato. Plant Biotechnol J 15: 14391452

Google Scholar: Author Only Title Only Author and Title

Phukela B, Geeta R, Das S, Tandon R (2020) Ancestral segmental duplication in Solanaceae is responsible for the origin of CRCaCRCb paralogues in the family. Mol Genet Genomics 295: 563-577

Google Scholar: Author Only Title Only Author and Title

R Development Core Team (2020) R: a language and environment for statistical computing: reference index. $R$ Foundation for Statistical Computing, Vienna, Austria. URL https://uww.r-project.org/

Google Scholar: Author Only Title Only Author and Title

Rodriguez-Leal D, Xu C, Kwon CT, Soyars C, Demesa-Arevalo E, Man J, Liu L, Lemmon ZH, Jones DS, Van Eck J, et al. (2019) Evolution of buffering in a genetic circuit controlling plant stem cell proliferation. Nat Genet 51: 786-792

Google Scholar: Author Only Title Only Author and Title

Rodríguez-Leal D, Lemmon ZH, Man J, Bartlett ME, Lippman ZB (2017) Engineering quantitative trait variation for crop improvement by genome editing. Cell 171: 470-480.e8

Google Scholar: Author Only Title Only Author and Title

Sablowski R (2007) Flowering and determinacy in Arabidopsis. J Exp Bot 58: 899-907

Google Scholar: Author Only Title Only Author and Title

Shang E, Ito T, Sun B (2019) Control of floral stem cell activity in Arabidopsis. Plant Signal Behav $14: 1659706$

Google Scholar: Author Only Title Only Author and Title 
Strable J, Wallace JG, Unger-Wallace E, Briggs S, Bradbury PJ, Buckler ES, Vollbrecht E (2017) Maize YABBY genes drooping leaf1 and drooping leaf2 regulate plant architecture. Plant Cell 29: 1622-1641

Google Scholar: Author Only Title Only Author and Title

Sun B, Ito T (2015) Regulation of floral stem cell termination in Arabidopsis. Front Plant Sci 6: 17

Google Scholar: Author Only Title Only Author and Title

Sun B, Zhou Y, Cai J, Shang E, Yamaguchi N, Xiao J, Looi LS, Wee WY, Gao X, Wagner D, et al. (2019) Integration of transcriptional repression and Polycomb-mediated silencing of WUSCHEL in floral meristems. Plant Cell 31: 1488-1505

Google Scholar: Author Only Title Only Author and Title

Sun B, Looi LS, Guo S, He Z, Gan ES, Huang J, Xu Y, Wee WY, Ito T (2014) Timing mechanism dependent on cell division is invoked by Polycomb eviction in plant stem cells. Science 343: 1248559

Google Scholar: Author Only Title Only Author and Title

Sun B, Xu Y, Ng KH, Ito T (2009) A timing mechanism for stem cell maintenance and differentiation in the Arabidopsis floral meristem Genes Dev 23: 1791-1804

Google Scholar: Author Only Title Only Author and Title

Sun W, Huang W, Li Z, Lv H, Huang H, Wang Y (2013) Characterization of a CRABS CLAW gene in basal eudicot species Epimedium sagittatum (Berberidaceae). Int J Mol Sci 14: 1119-1131

Google Scholar: Author Only Title Only Author and Title

The 100 Tomato Genome Sequencing Consortium (2014) Exploring genetic variation in the tomato (Solanum section Lycopersicon) clade by whole-genome sequencing. Plant J 80: 136-148

Google Scholar: Author Only Title Only Author and Title

Tian T, Liu Y, Yan H, You Q, Yi X, Du Z, Xu W, Su Z(2017) agriGO v2.0: a GO analysis toolkit for the agricultural community, 2017 update. Nucleic Acids Res 45: W122-W129

Google Scholar: Author Only Title Only Author and Title

van der Knaap E, Chakrabarti M, Chu YH, Clevenger JP, Illa-Berenguer E, Huang Z, Keyhaninejad N, Mu Q, Sun L, Wang Y, et al. (2014) What lies beyond the eye: the molecular mechanisms regulating tomato fruit weight and shape. Front Plant Sci 5: 227

Google Scholar: Author Only Title Only Author and Title

Vazquez-Vilar M, Bernabé-Orts JM, Fernandez-Del-Carmen A, Zarsolo P, Blanca J, Granell A, Orzaez D (2016) Amodular toolbox for gRNA-Cas9 genome engineering in plants based on the GoldenBraid standard. Plant Methods 12: 10

Google Scholar: Author Only Title Only Author and Title

Wang A, Tang J, Li D, Chen C, Zhao X, Zhu L (2009) Isolation and functional analysis of LiYAB1, a YABBY family gene, from lily (Lilium longiflorum). J Plant Physiol 166: 988-995

Google Scholar: Author Only Title Only Author and Title

Wesley SV, Helliwell CA, Smith NA, Wang MB, Rouse DT, Liu Q, Gooding PS, Singh SP, Abbott D, Stoutjesdijk PA, et al. (2001)

Construct design for efficient, effective and high-throughput gene silencing in plants. Plant J 27: 581-590

Google Scholar: Author Only Title Only Author and Title

Winer J, Jung CK, Shackel I, Williams PM (1999) Development and validation of real-time quantitative reverse transcriptasepolymerase chain reaction for monitoring gene expression in cardiac myocytes in vitro. Anal Biochem 270: 41-49

Google Scholar: Author Only Title Only Author and Title

Xu C, Liberatore KL, MacAlister CA, Huang Z, Chu YH, Jiang K, Brooks C, Ogawa-Ohnishi M, Xiong G, Pauly M, et al. (2015) Acascade of arabinosyltransferases controls shoot meristem size in tomato. Nat Genet 47: 784-792

Google Scholar: Author Only Title Only Author and Title

Yamada T, Yokota S, Hirayama Y, Imaichi R, Kato M, Gasser CS (2011) Ancestral expression patterns and evolutionary diversification of YABBY genes in angiosperms. Plant J 67: 26-36

Google Scholar: Author Only Title Only Author and Title

Yamaguchi N, Huang J, Tatsumi Y, Abe M, Sugano SS, Kojima M, Takebayashi Y, Kiba T, Yokoyama R, Nishitani K, et al. (2018)

Chromatin-mediated feed-forward auxin biosynthesis in floral meristem determinacy. Nat Commun 9: 5290

Google Scholar: Author Only Title Only Author and Title

Yamaguchi N, Huang J, Xu Y, Tanoi K, Ito T (2017) Fine-tuning of auxin homeostasis governs the transition from floral stem cell maintenance to gynoecium formation. Nat Commun 8: 1125

Google Scholar: Author Only Title Only Author and Title

Yamaguchi T, Nagasawa N, Kawasaki S, Matsuoka M, Nagato Y, Hirano HY (2004) The YABBY gene DROOPING LEAF regulates carpel specification and midrib development in Oryza sativa. Plant Cell 16: 500-509

Google Scholar: Author Only Title Only Author and Title

Yuste-Lisbona FJ, Jiménez-Gómez JM, Capel C, Lozano R (2021) Effective mapping by sequencing to isolate causal mutations in the tomato genome. Methods Mol Biol 2264: 89-103

Google Scholar: Author Only Title Only Author and Title 
Yuste-Lisbona FJ, Fernández-Lozano A, Pineda B, Bretones S, Ortíz-Atienza A, García-Sogo B, Müller NA, Angosto T, Capel J, Moreno $V$, et al. (2020) ENO regulates tomato fruit size through the floral meristem development network. Proc Natl Acad Sci U S A117: 81878195

Google Scholar: Author Only Title Only Author and Title 